\title{
Lattice of Finite Group Actions on Prism Manifolds
}

\author{
John E. Kalliongis ${ }^{1}$, Ryo Ohashi ${ }^{2}$ \\ ${ }^{1}$ Department of Mathematics and Computer Science, Saint Louis University, St. Louis, USA \\ ${ }^{2}$ Department of Mathematics, King's College, Wilkes-Barre, USA \\ Email: kalliongisje@slu.edu, ryoohashi@kings.edu
}

Received October 12, 2011; revised December 11, 2011; accepted December 22, 2011

\begin{abstract}
The set of finite group actions (up to equivalence) which operate on a prism manifold $M$, preserve a Heegaard Klein bottle and have a fixed orbifold quotient type, form a partially ordered set. We describe the partial ordering of these actions by relating them to certain sets of ordered pairs of integers. There are seven possible orbifold quotient types, and for any fixed quotient type we show that the partially ordered set is isomorphic to a union of distributive lattices of a certain type. We give necessary and sufficent conditions, for these partially ordered sets to be isomorphic and to be a union of Boolean algebras.
\end{abstract}

Keywords: Finite Group Action; Prism 3-Manifold; Equivalence of Actions; Orbifold; Partially Ordered Set; Distributive Lattice

\section{Introduction}

This paper examines the partially ordered sets consisting of equivalence classes of finite group actions acting on prism manifolds and having a fixed orbifold quotient type. For a fixed quotient type, we show that the partially ordered set is a union of distributive lattices of a certain type. These lattices have the structure of factorization lattices. The results in this paper relate to those in [1], where those authors study a family of orientation reversing actions on lens spaces which is partially ordered in terms of a subset of the lattice of Gaussian integers ordered by divisibility (see also [2]). Finite group actions on prism manifolds were also studied in [3].

Let $M$ be a prism manifold and let $G$ be a finite group. A $G$-action on $M$ is a monomorphism $\varphi: G \rightarrow \operatorname{Diff}(M)$ where $\operatorname{Diff}(M)$ is the group of self-diffeomorphisms of $M$. Two group actions $\varphi: G \rightarrow \operatorname{Diff}(M)$ and $\varphi^{\prime}: G^{\prime} \rightarrow$ $\operatorname{Diff}\left(M^{\prime}\right)$ are equivalent if there is a homeo-morphism $h: M \rightarrow M^{\prime}$ such that $\varphi^{\prime}\left(G^{\prime}\right)=h \circ \varphi(G) \circ h^{-1}$, and we let $[\varphi]$ denote the equivalence class. If $\varphi: G \rightarrow \operatorname{Diff}(M)$ is an action, let $v_{\varphi}: M \rightarrow M / \varphi$ be the orbifold covering map. The set of equivalence classes of actions on prism manifolds forms a partially ordered set by defining $\left[\varphi^{\prime}\right] \geq[\varphi]$ if there is a covering $v: M^{\prime} \rightarrow M$ such that $v_{\varphi^{\prime}}=v_{\varphi} \circ v$.

A prism manifold is defined as follows: Let $T=S^{1} \times S^{1}$ be a torus where $S^{1}=\{z \in \mathbb{C}:|z|=1\}$ is viewed as the set of complex numbers of norm 1 and $I=[0,1]$. The twisted $I$-bundle over a Klein bottle is the quotient space $W=T \times I /(u, v, t) \simeq(-u, \bar{v}, 1-t)$. Let $D^{2}$ be a unit disk with $\partial D^{2}=S^{1}$ and let $V=S^{1} \times D^{2}$ be a solid torus. Then the boundary of both $V$ and $W$ is a torus $S^{1} \times S^{1}$. For relatively prime integers $b$ and $d$, there exist integers $a$ and $b$ such that $a d-b c=-1$. The prism manifold $M(b, d)$ is obtained by identifying the boundary of $V$ to the boundary of $W$ by the homeomorphism $\psi: \partial V \rightarrow \partial W$ defined by $\psi(u, v)=\left(u^{a} v^{b}, u^{c} v^{d}\right)$ for $(u, v) \in \partial V=S^{1} \times$ $S^{1}$. The integers $b$ and $d$ determine $M(b, d)$, up to homeomorphism. An embedded Klein bottle $K$ in $M(b, d)$ is called a Heegaard Klein bottle if for any regular neighborhood $N(K)$ of $K, N(K)$ is a twisted $I$-bundle over $K$ and the closure of $M(b, d)-N(K)$ is a solid torus. Any $G$-action which leaves a Heegaard Klein bottle invariant is said to split.

We describe in Section 2, the $G$-actions (up to equivalence) which can act on a prism manifold and the seven possible quotient orbifolds $\mathcal{O}_{i}(\beta, \delta)$ for $1 \leq i \leq 7$ where $\beta$ and $\delta$ are some positive integers. For example, the orbifold $\mathcal{O}_{1}(\beta, \delta)$ is an orbifold whose underlying space is a prism manifold with a simple closed curve as an exceptional set of type $k=$ g.c.d $\{\beta, \delta\}$. The closure of the complement of the exceptional set is a twisted I-bundle over a Klein bottle. Section 3 gives necessary and sufficient conditions for an orbifold of type $\mathcal{O}_{i}(\beta, \delta)$ to be regularly covered by a prism manifold.

Let $\mathcal{L}^{i}(\beta, \delta)$ be the partially ordered set of equivalence classes of $G$-actions with orbifold quotient 
$\mathcal{O}_{i}(\beta, \delta)$. Define a set

$$
\begin{aligned}
& \mathcal{D}^{1}(\beta, \delta)=\left\{(b, d) \in \mathbb{Z}^{+} \times \mathbb{Z}^{+}: \text {g.c.d }\{b, d\}=1, b\right. \\
& \text { divides } \left.\beta, \frac{\beta}{b}=1(\bmod 2) \text {, and } d=\delta \text { or } 2 d=\delta\right\} .
\end{aligned}
$$

We show in Sections 4 and 6 that the set $\mathcal{D}^{1}(\beta, \delta)$ is a distributive lattice which is isomorphic as a partially ordered set to $\mathcal{L}^{\mathrm{l}}(\beta, \delta)$, and this implies that $\mathcal{L}^{\mathrm{l}}(\beta, \delta)$ is also a distributive lattice. For $2 \leq i \leq 7$, we show that $\mathcal{L}^{i}(\beta, \delta)$ is isomorphic as a partially ordered set to a union of lattices of type $\mathcal{D}^{1}(x, y)$. In addition, we give necessary and sufficient conditions for two lattices of type $\mathcal{D}^{1}(x, y)$ to be isomorphic.

A $G$-action is primitive if it does not contain a nontrivial normal subgroup which acts freely. These actions determine minimal elements in the partially ordered sets. We determine the primitive actions for each possible orbifold quotient in Section 5.

In Section 6 we compute the maximum length of a chain in the partially ordered sets $\mathcal{L}^{i}(\beta, \delta)$. Furthermore, if $b_{0}$ is the largest odd divisor of $\beta$ such that g.c.d $\left\{b_{0}, \delta\right\}=1$ and $b_{0}=\prod_{i=1}^{k} p_{i}^{l_{i}}$ is the prime decomposition, then we show that $\mathcal{D}^{1}(\beta, \delta)$ is a Boolean algebra if and only if $l_{i}=1$ for all $1 \leq i \leq k$.

When $\mathcal{O}_{1}(m, n)$ is a prism manifold, we consider in Section 7 a partially ordered set of non-cyclic subgroups $\mathcal{S}(m, n)$ of $\pi_{1}\left(\mathcal{O}_{1}(m, n)\right)$. We show that $\mathcal{S}(m, n)$ is a lattice isomorphic to $\mathcal{D}^{1}(m, n)$ where the partial ordering on the groups is given by $G_{2} \geq G_{1}$ if $G_{2}$ is a subgroup of $G_{1}$. The meet $G_{1} \wedge G_{2}=\left\langle G_{1}, G_{2}\right\rangle$ and the join $G_{1} \vee G_{2}=G_{1} \cap G_{2}$. Moreover we show that there exists a sublattice $\mathcal{A}$ of $\mathcal{S}(m, n)$ which is a Boolean algebra, and a lattice homomorphism $\mathcal{S}(m, n) \rightarrow \mathcal{A}$ which restricts to the identity on $\mathcal{A}$.

Section 8 is devoted to several examples which illustrate some of the main results.

\section{Actions on Prism Manifolds}

In this section we describe a set of $G$-actions on a prism manifold $M(b, d)$ which leave a Heegaard Klein bottle invariant and their quotient spaces $M(b, d) / G$. We obtain seven quotient types $\mathcal{O}_{i}(\beta, \delta)$ for $1 \leq i \leq 7$ where $\beta$ and $\delta$ are some positive integers. It follows by [4] that any $G$-action which leaves a Heegaard Klein bottle invariant is equivalent to one of the actions in Quotient type $[i]$ for some $1 \leq i \leq 7$, and $M(b, d) / G=\mathcal{O}_{i}(\beta, \delta)$. By [4] these actions are completely determined by their restriction to a Heegaard Klein bottle $K$. We begin by describing $G$-actions on $K$ and note that these actions extend to all of $M(b, d)$. We will list the actions by their quotient type.

Let $V(k)$ be the orbifold solid torus with exceptional set the core of type $k$ and let $B(k)=V(k) / \tau$ be the Conway ball, where $\tau: V(k) \rightarrow V(k)$ is the involution defined by $\tau(u, v)=(\bar{u}, \bar{v})$. The Conway sphere $\Sigma=\partial B(k)$ has 4 cone points, each of order 2 .

It is convenient to view the Klein bottle as the set of equivalence classes

$$
\begin{aligned}
K= & \left\{[r u]: 1 / 2 \leq r \leq 2, u \in S^{1},\right. \\
& \left.|u|=1,-\frac{1}{2} u \sim \frac{1}{2} u,-2 u \sim 2 u\right\} .
\end{aligned}
$$

1) Quotient type $\mathcal{O}_{1}(\beta, \delta)$. For $m=2 n+1$, define actions $\alpha_{1}: \mathbb{Z}_{m} \rightarrow \operatorname{Diff}(K)$ and $\beta_{1}: \mathbb{Z}_{2 m} \rightarrow \operatorname{Diff}(K)$ by $\alpha(1)([r u])=\left[r u e^{\frac{2 \pi i}{2 n+1}}\right]$ and $\beta_{1}(1)([r u])=\left[\frac{1}{r} u e^{\frac{\pi i}{2 n+1}}\right]$ where 1 represents a generator of the group. The quotients $K / \alpha_{1}$ and $K / \beta_{1}$ are both Klein bottles. These actions extend to the prism manifold $M(b, d)$ and we denote these extensions using the same letters to obtain $\alpha_{1}: \mathbb{Z}_{m} \rightarrow \operatorname{Diff}(M(b, d))$ and $\beta_{1}: \mathbb{Z}_{2 m} \rightarrow \operatorname{Diff}(M(b, d))$. The orbifold quotient for these actions is denoted by $\mathcal{O}_{1}(\beta, \delta)=V(k) \cup_{\psi} W_{1}$, where $W_{1}$ is a twisted I-bundle over the Klein bottle and $\psi: \partial V(k) \rightarrow \partial W_{i}$ is defined by $\psi(u, v)=\left(u^{\alpha} v^{\frac{\beta}{k}}, u^{\gamma} v^{\frac{\delta}{k}}\right)$ where $\beta$ and $\delta$ are integers, and $k=g \cdot c \cdot d\{\beta, \delta\}$. It follows that the quotient $M(b, d) / \alpha_{1}$ is the orbifold denoted by $\mathcal{O}_{1}(b(2 n+1), d)$ and the qoutient $M(b, d) / \beta_{1}$ is the orbifold denoted by $\mathcal{O}_{1}(b(2 n+1), 2 d)$.

2) Quotient type $\mathcal{O}_{2}(\beta, \delta)$. For $m=2 n$ define actions $\alpha_{2}: \mathbb{Z}_{m} \rightarrow \operatorname{Diff}(K)$ and $\beta_{2}: \mathbb{Z}_{2} \times \mathbb{Z}_{m} \rightarrow \operatorname{Diff}(K)$ by $\alpha_{2}(1)[r u]=\left[r u e^{\frac{\pi i}{n}}\right]$, and $\beta_{2}(1,0)([r u])=\left[\frac{1}{r} u\right]$ and $\beta_{2}(1,0)([r u])=\left[r u e^{\frac{\pi i}{n}}\right]$. The quotients $K / \alpha_{2}$ and $K / \beta_{2}$ are both mirrored annuli. These actions extend to all of $M(b, d)$ and we obtain $\alpha_{2}: \mathbb{Z}_{2 n} \rightarrow \operatorname{Diff}(M(b, d))$ and $\beta_{2}: \mathbb{Z}_{2} \times \mathbb{Z}_{2 n} \rightarrow \operatorname{Diff}(M(b, d))$. The orbifold quotient for these actions is denoted by $\mathcal{O}_{2}(\beta, \delta)=V(k) \cup_{\psi} W_{2}$, where $W_{2}=(T \times I) /(u, v, t) \simeq(u, \bar{v}, 1-t)$ is a twisted I-bundle over the mirrored annulus $m A$ and $\psi$ is defined as in Case 1. The orbifold quotient $M(b, d) / \alpha_{2}=\mathcal{O}_{2}(2 n b, d)$ and $M(b, d) / \beta_{2}=\mathcal{O}_{2}(2 n b, 2 d)$.

3) Quotient type $\mathcal{O}_{3}(\beta, \delta)$. Define $\alpha_{3}: \mathbb{Z}_{2} \times \mathbb{Z}_{2 n+1} \rightarrow$ $\operatorname{Diff}(K)$ and $\beta_{3}: \mathbb{Z}_{4 n} \rightarrow \operatorname{Diff}(K)$ by

$\alpha_{3}(1,0)([r u])=\left[\frac{1}{r} u\right], \quad \alpha_{3}(1,0)([r u])=\left[-r u e^{\frac{\pi i}{2 n+1}}\right]$, and 
$\beta_{3}(1)([r u])=\left[\frac{1}{r} u e^{\frac{\pi i}{2 n}}\right]$. The quotients $K / \alpha_{3}$ and $K / \beta_{3}$ are mirrored Möbius bands. These actions extend to the prism manifold $M(b, d)$ and we obtain $\alpha_{3}: \mathbb{Z}_{2} \times \mathbb{Z}_{2 n+1} \rightarrow$ $\operatorname{Diff}(M(b, d))$ and $\beta_{3}: \mathbb{Z}_{4 n} \rightarrow \operatorname{Diff}(M(b, d))$. The orbofold quotient for these actions is $\mathcal{O}_{3}(\beta, \delta)=V(k) \cup_{\psi} W_{3}$ $V(k) \cup_{\psi} W_{3}$ where $W_{3}=(T \times I) /(u, v, t) \simeq(v, u, 1-t)$ is a twisted $I$-bundle over the mirrored Möbius band $m M$. The orbifold quotient

$$
M(b, d) / \alpha_{3}=\mathcal{O}_{3}(b(2 n+1)+d, b(2 n+1)-d)
$$

and

$$
M(b, d) / \beta_{3}=\mathcal{O}_{3}(2 n b+d, 2 n b-d) .
$$

4) Quotient type $\mathcal{O}_{4}(\beta, \delta)$. For $m=2 n+1$, define the action $\alpha_{4}: \operatorname{Dih}\left(\mathbb{Z}_{2 n+1}\right) \rightarrow \operatorname{Diff}(K)$ by

$\alpha_{4}(1,0)([r u])=\left[-r u e^{\frac{\pi i}{2 n+1}}\right]$ and $\alpha_{4}(0,1)([r u])=\left[\frac{1}{r} \bar{u}\right]$.

The quotient $K / \alpha_{4}$ is the projective plane $P^{2}(2,2)$ containing two cone points of order two. This action extends to $M(b, d)$ and we obtain $\alpha_{4}: \operatorname{Dih}\left(\mathbb{Z}_{2 n+1}\right) \rightarrow$ $\operatorname{Diff}(M(b, d))$. The orbifold quotient is $\mathcal{O}_{4}(\beta, \delta)=$ $B(k) \cup_{\bar{\psi}} W_{4}$ where $W_{4}=\Sigma \times I /(z, t) \simeq(-z, 1-t)$ is the twisted $I$-bundle over $P^{2}(2,2)$ and $\bar{\psi}$ is the homeomorphism of $\Sigma$ induced by $\psi$. The orbifold quotient $M(b, d) / \alpha_{4}=\mathcal{O}_{4}(b(2 n+1), d)$.

5) Quotient type $\mathcal{O}_{5}(\beta, \delta)$. Define the following actions: $\alpha_{5}: \operatorname{Dih}\left(\mathbb{Z}_{2 n+1}\right) \rightarrow \operatorname{Diff}(K)$ where

$\alpha_{5}(1,0)([r u])=\left[-r u e^{\frac{\pi i}{2 n+1}}\right]$ and $\alpha_{5}(0,1)[r u]=[r \bar{u}]$;

$\beta_{5}: \operatorname{Dih}\left(\mathbb{Z}_{4 n+2}\right) \rightarrow \operatorname{Diff}(K)$ where

$\beta_{5}(1,0)([r u])=\left[\frac{1}{r} u e^{\frac{\pi i}{2 n+1}}\right]$ and $\beta_{5}(0,1)([r u])=[r \bar{u}]$;

$\gamma_{5}: \operatorname{Dih}\left(\mathbb{Z}_{4 n+2}\right) \rightarrow \operatorname{Diff}(K)$ where

$\gamma_{5}(1,0)([r u])=\left[r u e^{\frac{2 \pi i}{4 n+2}}\right]$ and $\gamma_{5}(0,1)([r u])=\left[\frac{1}{r} \bar{u}\right]$; and $\delta_{5}: \operatorname{Dih}\left(\mathbb{Z}_{4 n}\right) \rightarrow \operatorname{Diff}(K)$ where

$\delta_{5}(1,0)([r u])=\left[r u e^{\frac{\pi i}{2 n}}\right]$ and $\delta_{5}(0,1)([r u])=\left[\frac{1}{r} \bar{u}\right]$. The

orbifold quotient for all these actions is the mirrored disk $\mathbb{D}^{2}(2,2)$. All these actions extend to $\operatorname{Diff}(M(b, d))$. If $W_{5}=\Sigma \times I /(z, t) \simeq(r(z), 1-t)$, where $r$ is a reflection exchanging a pair of cone points, is the twisted I-bundle over $\mathbb{D}^{2}(2,2)$, then the orbifold quotient for these extended actions is $\mathcal{O}_{5}(\beta, \delta)=B(k) \cup_{\bar{\psi}} W_{5}$. We obtain

$$
\begin{aligned}
& M(b, d) / \alpha_{5}=\mathcal{O}_{5}(b(2 n+1), d), \\
& M(b, d) / \beta_{3}=\mathcal{O}_{5}(b(2 n+1), 2 d),
\end{aligned}
$$

$$
M(b, d) / \gamma_{5}=\mathcal{O}_{5}(b(4 n+2), d)
$$

and

$$
M(b, d) / \delta_{5}=\mathcal{O}_{5}(4 n b, d) .
$$

6) Quotient type $\mathcal{O}_{6}(\beta, \delta)$. Define actions $\alpha_{6}, \beta_{6}$ : $\operatorname{Dih}\left(\mathbb{Z}_{2 n}\right) \rightarrow \operatorname{Diff}(K)$ as follows:

$\alpha_{6}(1,0)([r u])=\left[\frac{1}{r} u e^{\frac{\pi i}{n}}\right]$ and $\alpha_{6}(0,1)([r u])=[r \bar{u}]$ if $n$ is even, and $\beta_{6}(1,0)([r u])=\left[\frac{1}{r} u e^{\frac{2 \pi i}{n}}\right]$ and $\beta_{6}(0,1)([r u])=\left[\frac{1}{r} \bar{u}\right]$ if $n$ is odd. The quotients $K / \alpha_{5}$ and $K / \beta_{5}$ are both a mirrored disk $\mathcal{O}=\mathbb{D}^{2}(2)$ containig a cone point of order two and two cone points of order two on the mirror. These actions extend to the prism manifold $M(b, d)$ and we obtain $\alpha_{6}, \beta_{6}: \operatorname{Dih}\left(\mathbb{Z}_{2 n}\right) \rightarrow$ $\operatorname{Diff}(M(b, d))$. The orbifold quotient for these actions is denoted by $\mathcal{O}_{6}(\beta, \delta)=B(k) \cup_{\bar{\psi}} W_{6}$ where

$W_{6}=\Sigma \times I /(z, t) \simeq(r(z), 1-t)$ the twisted $I$-bundle over the mirrored disk $\mathbb{D}^{2}(2)$, and $r$ is a reflection leaving two cone points fixed and exchanging the other two cone points. The orbifold quotients $M(b, d) / \alpha_{5}$ and $M(b, d) / \beta_{6}$ are both $\mathcal{O}_{6}(b n+d, b n-d)$.

7) Quotient type $\mathcal{O}_{7}(\beta, \delta)$. Define $\alpha_{7}: \operatorname{Dih}\left(\mathbb{Z}_{2 n}\right) \rightarrow$ $\operatorname{Diff}(K)$ and $\beta_{7}: \operatorname{Dih}\left(\mathbb{Z}_{2} \times \mathbb{Z}_{2 n}\right) \rightarrow \operatorname{Diff}(K)$ as follows: $\alpha_{7}(1,0)([r u])=\left[r u e^{\frac{\pi i}{n}}\right], \alpha_{7}(0,1)([r u])=[r \bar{u}]$, and $\beta_{7}(1,0,0)([r u])=\left[-\frac{1}{r} u\right], \beta_{7}(0,1,0)([r u])=\left[r u e^{\frac{\pi i}{n}}\right]$, and $\beta_{7}(0,0,1)([r u])=[r \bar{u}]$. The quotients $K / \alpha_{7}$ and $K / \beta_{7}$ are both a mirrored disk $\mathcal{O}=\mathbb{D}^{2}(0)$ containig four cone points of order two on the mirror. These actions extend to the prism manifold $M(b, d)$ and we obtain

$$
\alpha_{7}: \operatorname{Dih}\left(\mathbb{Z}_{2 n}\right) \rightarrow \operatorname{Diff}(M(b, d))
$$

and

$$
\beta_{7}: \operatorname{Dih}\left(\mathbb{Z}_{2} \times \mathbb{Z}_{2 n}\right) \rightarrow \operatorname{Diff}(M(b, d)) .
$$

If $W_{7}=\Sigma \times I /(z, t) \simeq(r(z), 1-t)$, where $r$ is a reflection leaving each cone points fixed, then $W_{7}$ is a twisted $I$-bundle over the mirrored disk $\mathbb{D}^{2}(0)$. The orbifold quotient for these extended actions is $\mathcal{O}_{6}(\beta, \delta)=$ $B(k) \cup_{\bar{\psi}} W_{6}$. We obtain $M(b, d) / \alpha_{7}=\mathcal{O}_{7}(2 n b, d)$ and $M(b, d) / \beta_{7}=\mathcal{O}_{7}(2 n b, 2 d)$.

\section{Prism Manifold Covers of Orbifolds}

In this section we give necessary and sufficient condi- 
tions for when the orbifold $\mathcal{O}_{i}(\beta, \delta), 1 \leq i \leq 7$, is covered by a prism manifold. The proofs rely on Section 2 .

Proposition 1. For the orbifold $\mathcal{O}_{1}(\beta, \delta)$, there exists a prism manifold cover if and only if either $\beta$ is odd or $\delta \neq 0 \quad(\bmod 4)$.

Proof. Suppose $\beta$ is odd. Then there exists a $\mathbb{Z}_{\beta}$-action on $M(1, \delta)$ such that

$$
M(1, \delta) \rightarrow M(1, \delta) / \mathbb{Z}_{\beta}=\mathcal{O}_{1}(\beta, \delta) .
$$

If $\beta$ is even, write $\beta=2^{l} \beta_{0}$ where $\beta_{0}$ is odd. If $\delta$ is odd, then there exists a $\mathbb{Z}_{\beta_{0}}$-action on $M\left(2^{l}, \delta\right)$ such that

$$
M\left(2^{l}, \delta\right) \rightarrow M\left(2^{l}, \delta\right) / \mathbb{Z}_{\beta_{0}}=\mathcal{O}_{1}(\beta, \delta) .
$$

Suppose now that $\beta$ and $\delta$ are both even where $\delta \neq 0$ $(\bmod 4)$. Write $\delta=2 \delta_{0}$ and $\beta=2^{m} \beta_{0}$ where $\delta_{0}$ and $\beta_{0}$ are both odd. Then there exists a $\mathbb{Z}_{2 \beta_{0}}$-action on $M\left(2^{m}, \delta_{0}\right)$ such that

$$
M\left(2^{m}, \delta_{0}\right) \rightarrow M\left(2^{m}, \delta_{0}\right) / \mathbb{Z}_{2 \beta_{0}}=\mathcal{O}_{1}\left(2^{m} \beta_{0}, 2 \delta_{0}\right) .
$$

For the converse, suppose that $\beta$ and $\delta$ are both even and there is a covering $M(b, d) \rightarrow \mathcal{O}_{i}(\beta, \delta)$. Then either $b(2 n+1)=\beta$ and $d=\delta$, or $b(2 n+1)=\beta$ and $2 d=\delta$. Since $\beta$ is even, it follows that 2 divides $b$. In the first case, 2 would also divide $d$, contradicting the fact that $b$ and $d$ are relatively prime. If $\delta \neq 0(\bmod 4)$, then again 2 divides $d$ giving a contradiction.

Proposition 2. For the orbifold $\mathcal{O}_{2}(\beta, \delta)$, there exists a prism manifold cover if and only if $\beta=0(\bmod 2)$.

Proof. Suppose that $\beta=0(\bmod 2)$. Write $\beta=2 \beta_{0}$. Then there exists a $\mathbb{Z}_{2 \beta_{0}}$-action on $M(1, \delta)$ such that

$$
M(1, \delta) \rightarrow M(1, \delta) / \mathbb{Z}_{2 \beta_{0}}=\mathcal{O}_{2}(\beta, \delta) .
$$

For the converse, suppose that $M(b, d) \rightarrow \mathcal{O}_{2}(\beta, \delta)$. Then either $2 n b=\beta$ and $d=\delta$, or $2 n b=\beta$ and $2 d=\delta$.

Proposition 3. For the orbifold $\mathcal{O}_{3}(\beta, \delta)$, there exists a prism manifold cover if and only if $\beta=\delta(\bmod 2)$.

Proof. Suppose that $\beta=\delta(\bmod 2)$ and let $d=\frac{\beta-\delta}{2}$. Suppose that $\beta+\delta=0(\bmod 4)$, and thus there exists an integer $n$ such that $\beta+\delta=4 n$. There exists a $\mathbb{Z}_{4 n}$ action on $M(1, d)$ such that

$$
\begin{aligned}
M(1, d) & \rightarrow M(1, d) / \mathbb{Z}_{4 n}=\mathcal{O}_{3}(2 n+d, 2 n-d) \\
& =\mathcal{O}_{3}(\beta, \delta) .
\end{aligned}
$$

If $\beta+\delta \neq 0(\bmod 4)$, then write $\frac{\beta+\delta}{2}=2 n+1$ for some $n$. There exists a $\mathbb{Z}_{2(2 n+1)}$-action on $M(1, d)$ such that

$$
\begin{aligned}
M(1, d) & \rightarrow M(1, d) / \mathbb{Z}_{2(2 n+1)}=\mathcal{O}_{3}(2 n+1+d, 2 n+1-d) \\
& =\mathcal{O}_{3}(\beta, \delta) .
\end{aligned}
$$

For the converse, suppose that $M(b, d) \rightarrow \mathcal{O}_{3}(\beta, \delta)$.
Then either $b(2 n+1)+d=\beta$ and $b(2 n+1)-d=\delta$, or $2 n b+d=\beta$ and $2 n b-d=\delta$ for some $n$. Subtracting the two equations in both cases, we obtain $2 d=\beta-\delta$.

Proposition 4. For the orbifold $\mathcal{O}_{4}(\beta, \delta)$, there exists a prism manifold cover if and only if either $\beta$ is odd or $\delta$ is odd.

Proof. Since $M(b, d) / \alpha_{1}$ always double covers $M(b, d) / \alpha_{4}$, using a proof similar to that in Proposition 1 shows that there is a prism manifold covering of $\mathcal{O}_{4}(\beta, \delta)$ if and only if $\beta$ or $\delta$ is odd by [4].

Proposition 5. A prism manifold covering for the orbifold $\mathcal{O}_{5}(\beta, \delta)$ always exists.

Proof. Suppose $\beta$ is an odd number. Then $M(1, \delta)$ admits a $\operatorname{Dih}\left(\mathbb{Z}_{\beta}\right)$-action whose quotient is $\mathcal{O}_{5}(\beta, \delta)$. If $\beta$ is even, we write $\beta=2^{m} \beta_{0}$ where $m \geq 1, \delta=2^{n} \delta_{0}$ where $n \geq 0$, and $\beta_{0}$ and $\delta_{0}$ are both odd numbers. If $n=0$ or $n=1$, then $M\left(2^{m}, \delta\right)$ and $M\left(2^{m}, \delta_{0}\right)$ admit $\operatorname{Dih}\left(\mathbb{Z}_{\beta_{0}}\right)$ and $\operatorname{Dih}\left(\mathbb{Z}_{2 \beta_{0}}\right)$-actions respectively, whose quotient space is $\mathcal{O}_{5}(\beta, \delta)$. If $n$ and $m$ are both greater than 1 , or if $m=1$ and $n \geq 2$, then $M(1, \delta)$ admits a $\operatorname{Dih}\left(\mathbb{Z}_{4\left(2^{m-1} \beta_{0}\right)}\right)$ or a $\operatorname{Dih}\left(\mathbb{Z}_{2 \beta_{0}}\right)$-action respectively, whose quotient space is $\mathcal{O}_{5}(\beta, \delta)$.

Proposition 6. For the orbifold $\mathcal{O}_{6}(\beta, \delta)$, there exists a prism manifold cover if and only if $\beta=\delta(\bmod 2)$.

Proof. Since $M(b, d) / \beta_{3}$ double covers $M(b, d) / \alpha_{6}$ and $M(b, d) / \alpha_{3}$ double covers $M(b, d) / \beta_{6}$, the result follows by Proposition 3.

Proposition 7. For the orbifold $\mathcal{O}_{7}(\beta, \delta)$, there exists a prism manifold cover if and only if $\beta=0(\bmod 2)$.

Proof. Since $M(b, d) / \alpha_{2}$ double covers $M(b, d) / \alpha_{7}$ and $M(b, d) / \beta_{2}$ double covers $M(b, d) / \beta_{7}$, the result follows by Proposition 2 .

\section{Poset of Actions on Prism Manifolds}

Recall that two group actions $\varphi: G \rightarrow \operatorname{Diff}(M)$ and $\varphi^{\prime}: G^{\prime} \rightarrow \operatorname{Diff}\left(M^{\prime}\right)$ are equivalent if there is a homeomorphism $h: M \rightarrow M^{\prime}$ such that $\varphi^{\prime}: G^{\prime}=h \circ \varphi(G) \circ h^{-1}$. If $\varphi: G \rightarrow \operatorname{Diff}(M)$ is an action, let $v_{\varphi}: M \rightarrow M / \varphi$ be the orbifold covering map.

Let $\mathcal{L}$ be the set of equivalence classes of actions on prisim manifolds which leave a Heegaard Klein bottle invariant. Now $\mathcal{L}$ is partially ordered by setting $\left[\varphi^{\prime}\right] \geq[\varphi]$ if there is a covering $v: M^{\prime} \rightarrow M$ such that $v_{\varphi^{\prime}}=v_{\varphi} \circ v$. Note that the covering $v: M^{\prime} \rightarrow M$ is also a regular covering.

For a pair of positive integers $\beta$ and $\delta$ let $\mathcal{L}^{\mathrm{l}}(\beta, \delta)$ denote the equivalence classes of those actions whose quotient type is $\mathcal{L}^{1}(\beta, \delta)$. Note that by Proposition 1 the set $\mathcal{L}^{\mathrm{l}}(\beta, \delta)$ is nonempty if and only if either $\beta$ is odd, or $\delta \neq 0 \quad(\bmod 4)$. Unless otherwise stated, we assume from now on that $\beta$ and $\delta$ are integers where either $\beta$ 
is odd or $\delta \neq 0(\bmod 4)$.

Let

$$
\mathcal{D}^{1}(\beta, \delta)=\left\{\{b, d\} \in Z^{+} \times Z^{+}: \operatorname{g.c.d}\{b, d\}=1,\right.
$$

$b$ divides $\beta, \frac{\beta}{b}=1(\bmod 2)$ and $d=\delta$, or $\left.2 d=\delta\right\}$.

It follows that $\mathcal{D}^{1}(\beta, \delta)$ is a partially ordered set under the ordering $\left(b_{2}, d_{2}\right) \geq\left(b_{1}, d_{1}\right)$ if $b_{2} \mid b_{1}$ and $d_{2} \mid d_{1}$. Let $\mathcal{D}_{*}^{1}(\beta, \delta)$ be the subset of $\mathcal{D}^{1}(\beta, \delta)$ consisting of all ordered pairs $(b, d) \in \mathcal{D}^{1}(\beta, \delta)$ where $d=\delta$. Note that $\mathcal{D}_{*}^{1}(\beta, \delta)=\mathcal{D}^{1}(\beta, \delta)$ if $\delta$ is odd. Moreover, if $\frac{\delta}{2} \neq 0$ $(\bmod 2)$ and $\beta$ is even, then $\mathcal{D}^{1}(\beta, \delta)=\mathcal{D}^{1}(\beta, \delta / 2)$.

Proposition 8. Let $\left(b_{1}, d_{1}\right)$ and $\left(b_{2}, d_{2}\right)$ be elements of the poset $\mathcal{D}^{1}(\beta, \delta)$. There exists elements $(b, d)$ and $\left(b^{\prime}, d^{\prime}\right)$ in $\mathcal{D}^{1}(\beta, \delta)$, such that $(b, d) \geq\left(b_{1}, d_{1}\right)$ and $(b, d) \geq\left(b_{2}, d_{2}\right)$, and $\left(b^{\prime}, d^{\prime}\right) \leq\left(b_{1}, d_{1}\right)$ and $\left(b^{\prime}, d^{\prime}\right) \leq$ $\left(b_{2}, d_{2}\right)$.

Proof. Let $b=$ g.c.d $\left\{b_{1}, b_{2}\right\}$. Note that since $d_{i}=\delta$ or $\delta / 2$ for $i=1,2$, it follows that if $d=\min \left\{d_{1}, d_{2}\right\}$, then $d \mid d_{i}$. Thus $b$ divides $\beta$ and $d$ is $\delta$ or $\delta / 2$. If $\beta / b$ is even, then it follows that 2 divides both $b_{1} / b$ and $b_{2} / b$, contradicting $b=$ g.c.d $\left\{b_{1}, b_{2}\right\}$. Thus $\beta / b$ is odd showing $(b, d) \in \mathcal{D}^{1}(\beta, \delta)$. Moeover $(b, d) \geq\left(b_{1}, d_{1}\right)$ and $(b, d) \geq\left(b_{2}, d_{2}\right)$. Let $b^{\prime}=$ l.c. $m\left\{b_{1}, b_{2}\right\}$ and $d^{\prime}=$ $\max \left\{d_{1}, d_{2}\right\}$. It follows that $\beta / b^{\prime}$ is odd, and hence $\left(b^{\prime}, d^{\prime}\right) \in \mathcal{D}^{1}(\beta, \delta)$. Furthermore $\left(b^{\prime}, d^{\prime}\right) \leq\left(b_{1}, d_{1}\right)$ and $\left(b^{\prime}, d^{\prime}\right) \leq\left(b_{2}, d_{2}\right)$.

Corollary 9. $\mathcal{D}^{1}(\beta, \delta)$ is a lattice where for $\left(b_{1}, d_{1}\right)$ and $\left(b_{2}, d_{2}\right)$ in $\mathcal{D}^{1}(\beta, \delta)$ the join

$$
\left(b_{1}, d_{1}\right) \vee\left(b_{2}, d_{2}\right)=\left(\text { g.c.d }\left\{b_{1}, b_{2}\right\}, \min \left\{d_{1}, d_{2}\right\}\right),
$$

and the meet

$$
\left(b_{1}, d_{1}\right) \wedge\left(b_{2}, d_{2}\right)=\left(\text { l.c.m }\left\{b_{1}, b_{2}\right\}, \max \left\{d_{1}, d_{2}\right\}\right) .
$$

Furthermore, $\mathcal{D}_{*}^{1}(\beta, \delta)$ is a sublattice of $\mathcal{D}^{1}(\beta, \delta)$.

Proposition 10. Let $\left(b_{1}, d_{1}\right)$ and $\left(b_{2}, d_{2}\right)$ be elements of $\mathcal{D}^{1}(\beta, \delta)$ such that $\left(b_{2}, d_{2}\right) \geq\left(b_{1}, d_{1}\right)$. Then there exists either a standard $\mathbb{Z}_{m}$-action $\alpha_{1}$ on $M\left(b_{2}, d_{2}\right)$, or a standard $\mathbb{Z}_{2 m}$-action $\beta_{1}$ on $M\left(b_{2}, d_{2}\right)$, which we denote by $\theta$, and a regular covering

$$
v_{\theta}: M\left(b_{2}, d_{2}\right) \rightarrow M\left(b_{2}, d_{2}\right) / \theta=M\left(b_{1}, d_{1}\right) .
$$

Proof. If $\left(b_{2}, d_{2}\right) \geq\left(b_{1}, d_{1}\right)$, then $b_{2} \mid b_{1}$ and $d_{2} \mid d_{1}$. Furthermore $d_{1}=\delta$, or $2 d_{1}=\delta$ and $d_{2}=\delta$, or $2 d_{2}=\delta$. Now $\frac{b_{1}}{b_{2}}=m, \beta=b_{1}\left(2 n_{1}+1\right)$, and $\beta=b_{2}\left(2 n_{1}+1\right)$ for some integers $m, n_{1}$ and $n_{2}$. Since $b_{1}\left(2 n_{1}+1\right)=b_{2}\left(2 n_{2}+1\right)$, it follows that $\left(2 n_{2}+1\right)=m\left(2 n_{1}+1\right)$, and therefore $m$ must be odd. Since $d_{2} \mid d_{1}$, the only possibilities are $d_{1}=d_{2}$ or $2 d_{2}=d_{1}$. If $d_{1}=d_{2}$, then there exists a
$\mathbb{Z}_{m}$-action $\alpha_{1}$ on $M\left(b_{2}, d_{2}\right)$ such that

$$
M\left(b_{2}, d_{2}\right) \rightarrow M\left(b_{2}, d_{2}\right) / \alpha_{1}=M\left(b_{1}, d_{1}\right) .
$$

If $2 d_{2}=d_{1}$, then there exists a $\mathbb{Z}_{2 m}$-action $\beta_{1}$ on $M\left(b_{2}, d_{2}\right)$ such that

$$
M\left(b_{2}, d_{2}\right) \rightarrow M\left(b_{2}, d_{2}\right) / \beta_{1}=M\left(b_{1}, d_{1}\right) .
$$

Proposition 11. Let $(b, d)$ be an element of $\mathcal{D}^{1}(\beta, \delta)$. Then there exists either a standard $\mathbb{Z}_{2 n+1}$-action $\alpha_{1}$ on $M(b, d)$, or a standard $\mathbb{Z}_{2(2 n+1)}$-action $\beta_{1}$ on $M(b, d)$ which we denote by $\varphi$, and a regular covering

$$
v_{\varphi}: M(b, d) \rightarrow M(b, d) / \varphi=\mathcal{O}_{1}(\beta, \delta) .
$$

Proof. Write $\frac{\beta}{b}=2 n+1$. If $d=\delta$, then there is a $\mathbb{Z}_{2 n+1}$-action $\alpha_{1}$ such that

$$
M(b, d) \rightarrow M(b, d) / \alpha_{1}=\mathcal{O}_{1}(\beta, \delta) .
$$

If $2 d=\delta$, then there is a $\mathbb{Z}_{2(2 n+1)}$-action $\beta_{1}$ on $M(b, d)$ such that

$$
M(b, d) \rightarrow M(b, d) / \beta_{1}=\mathcal{O}_{1}(\beta, \delta) .
$$

Theorem 12. For each pair of positive integers $\beta$ and $\delta$, the poset $\mathcal{L}^{\mathbb{1}}(\beta, \delta)$ is isomorphic to the poset $\mathcal{D}^{1}(\beta, \delta)$.

Proof. Define a function $f: \mathcal{D}^{1}(\beta, \delta) \rightarrow \mathcal{L}^{1}(\beta, \delta)$ as follows: let $(b, d) \in \mathcal{D}^{1}(\beta, \delta)$. There exists either a standard $\mathbb{Z}_{\frac{\beta}{b}}$-action if $d=\delta$, or a standard $\mathbb{Z}_{2\left(\frac{\beta}{b}\right)}$-action if $2 d=\delta$ on $M(b, d)$, which we denote by $\varphi$, such that

$$
M(b, d) \rightarrow M(b, d) / \varphi=\mathcal{O}_{1}(\beta, \delta) .
$$

Define $f(b, d)=[\varphi] \in \mathcal{L}^{1}(\beta, \delta)$.

Suppose $f\left(b_{1}, d_{1}\right)=\left[\varphi_{1}\right]=\left[\varphi_{2}\right]=f\left(b_{2}, d_{2}\right)$. Since $\varphi_{1}$ and $\varphi_{2}$ are equivalent, there exists a homeomorphism $h: M\left(b_{1}, d_{1}\right) \rightarrow M\left(b_{2}, d_{2}\right)$ such that

$\varphi_{1}\left(G^{\prime}\right)=h \circ \varphi_{2}(G) \circ h^{-1}$. Since $M\left(b_{1}, d_{1}\right)$ and $M\left(b_{2}, d_{2}\right)$ are homeomorphic, it follows that $b_{1}=b_{2}$ and $d_{1}=d_{2}$, showing $f$ is one-to-one.

Let $[\eta] \in \mathcal{L}^{1}(\beta, \delta)$. Then there exist a prism manifold $M(b, d)$ such that

$$
v_{\eta}: M(b, d) \rightarrow M(b, d) / \eta=\mathcal{O}_{1}(\beta, \delta) .
$$

We may assume that $b$ and $d$ are both positive. By [4], $\eta$ is equivalent to one of the standard actions $\alpha_{1}$ or $\beta_{1}$, and $M(b, d) / \eta=\mathcal{O}_{1}(b(2 n+1), d)$ or $\mathcal{O}_{1}(b(2 n+1), 2 d)$ respectively, for some positive integer $n$. Therefore $\frac{\beta}{b}=1(\bmod 2)$ and $d=\delta$ or $2 d=\delta$. If $\varphi$ is either $\alpha_{1}$ or $\beta_{1}$, then $f(b, d)=[\varphi]=[\eta]$, showing $f$ is onto.

Suppose now that $\left(b_{2}, d_{2}\right) \geq\left(b_{1}, d_{1}\right)$. Let $f\left(b_{1}, d_{1}\right)=\left[\varphi_{1}\right]$ and $f\left(b_{2}, d_{2}\right)=\left[\varphi_{2}\right]$ where $\varphi_{1}$ and $\varphi_{2}$ are the standard 
$\mathbb{Z}_{\varepsilon_{1}\left(\beta / b_{1}\right)}$ and $\mathbb{Z}_{\varepsilon_{2}\left(\beta / b_{2}\right)}$-actions respectively on $M\left(b_{1}, d_{1}\right)$ and $M\left(b_{2}, d_{2}\right)$ and $\varepsilon_{i}=1$ or 2 . We have the coverings

$$
v_{\varphi_{1}}: M\left(b_{1}, d_{1}\right) \rightarrow M\left(b_{1}, d_{1}\right) / \varphi_{1}=\mathcal{O}_{1}(\beta, \delta)
$$

and

$$
v_{\varphi_{1}}: M\left(b_{1}, d_{1}\right) \rightarrow M\left(b_{1}, d_{1}\right) / \varphi_{1}=\mathcal{O}_{1}(\beta, \delta) .
$$

By Proposition 10 there is a standard $\mathbb{Z}_{\varepsilon\left(b_{1} / b_{2}\right)}$-action $\theta$ on $M\left(b_{2}, d_{2}\right)$ where $\varepsilon=1$ or 2 , and a regular covering covering

$$
v_{\theta}: M\left(b_{2}, d_{2}\right) \rightarrow M\left(b_{2}, d_{2}\right) / \theta=M\left(b_{1}, d_{1}\right) .
$$

Since these are standard actions and $\frac{\beta / b_{2}}{b_{1} / b_{2}}=\frac{\beta}{b_{1}}$ it follows that $v_{\varphi_{2}}=v_{\varphi_{1}} \circ v_{\theta}$. This shows that $\left[\varphi_{2}\right] \geq\left[\varphi_{1}\right]$.

Corollary 13. $\mathcal{L}^{\mathrm{l}}(\beta, \delta)$ is a lattice.

We will now consider maximal and minimal elements in $\mathcal{D}^{1}(\beta, \delta)$. Write $\beta=2^{n} \beta_{0}$ where $\beta_{0}$ is odd. Then the maximal element in $\mathcal{D}^{1}(\beta, \delta)$ is $\left(2^{n}, \delta\right)$ if $\delta$ is odd, and $\left(2^{n}, \delta / 2\right)$ if $\delta$ is even. Note that if $(b, d) \in$ $\mathcal{D}^{1}(\beta, \delta)$, then $2^{n}$ divides $b$ and $\frac{b}{2^{n}}$ is odd. In describing the minimal elements let $b_{0}$ be the largest odd divisor of $\beta_{0}$ such that g.c.d $\left\{b_{0}, \delta\right\}=1$. If $\delta$ is odd or if $n=0$, then the minimal element in $\mathcal{D}^{1}(\beta, \delta)$ is $\left(2^{n} b_{0}, \delta\right)$, otherwise the minimal element is $\left(2^{n} b_{0}, \delta / 2\right)$.

We say an element $\left(b_{1}, d_{1}\right)$ is directly below $\left(b_{2}, d_{2}\right)$ or that $\left(b_{2}, d_{2}\right)$ is directly above $\left(b_{1}, d_{1}\right)$ if whenever $\left(b_{1}, d_{1}\right) \leq(b, d) \leq\left(b_{2}, d_{2}\right)$, then either $\left(b_{1}, d_{1}\right)=(b, d)$ or $(b, d)=\left(b_{2}, d_{2}\right)$.

Theorem 14. Let $\left(2^{m_{0}} b_{0}, \delta_{1} / \varepsilon_{1}\right)$ and $\left(2^{n_{0}} c_{0}, \delta_{2} / \varepsilon_{2}\right)$ be the minimal elements in $\mathcal{D}^{1}\left(\beta_{1}, \delta_{1}\right)$ and $\mathcal{D}^{1}\left(\beta_{2}, \delta_{2}\right)$ respectively where $\varepsilon_{i}=1$ or 2 , and let $b_{0}=\prod_{i=1}^{k} p_{i}^{m_{i}}$ and $c_{0}=\prod_{i=1}^{s} q_{i}^{n_{i}}$ be the prime decompositions. Suppose one of the following holds:

1) $\delta_{1}$ and $\delta_{2}$ are both odd.

2) $\delta_{1}$ and $\delta_{2}$ are both even and $m_{0} n_{0} \neq 0$.

3) $\delta_{1}$ and $\delta_{2}$ are both even and $m_{0}=n_{0}=0$.

4) $\delta_{1}$ even with $m_{0} \neq 0$ and $\delta_{2}$ odd.

Then $\mathcal{D}^{1}\left(\beta_{1}, \delta_{1}\right)$ is isomorphic to $\mathcal{D}^{1}\left(\beta_{2}, \delta_{2}\right)$ if and only if $k=s$ and after reordering $m_{i}=n_{i}$ for $i=1, \cdots, k$.

If $\delta_{1}$ is odd and $\delta_{2}$ is even with $n_{0}=0$, then $\mathcal{D}^{1}\left(\beta_{1}, \delta_{1}\right)$ is isomorphic to $\mathcal{D}^{1}\left(\beta_{2}, \delta_{2}\right)$ if and only if $k=s+1$, after reordering $m_{i}=n_{i}$ for $i=1,2, \cdots, k-1$, and $m_{k}=1$.

Proof. We will first assume that $\delta_{1}$ and $\delta_{2}$ are both odd. Suppose $f: \mathcal{D}^{1}\left(\beta_{1}, \delta_{1}\right) \rightarrow \mathcal{D}^{1}\left(\beta_{2}, \delta_{2}\right)$ is an isomorphism. Now $\left(2^{m_{0}}, \delta_{1}\right)$ and $\left(2^{n_{0}}, \delta_{2}\right)$ are the maximal elements of $\mathcal{D}^{1}\left(\beta_{1}, \delta_{1}\right)$ and $\mathcal{D}^{1}\left(\beta_{2}, \delta_{2}\right)$ respectively, and $f\left(2^{m_{0}}, \delta_{1}\right)=\left(2^{n_{0}}, \delta_{2}\right)$. The elements directly below $\left(2^{m_{0}}, \delta_{1}\right)$ in $\mathcal{D}^{1}\left(\beta_{1}, \delta_{1}\right)$ are $\left(2^{m_{0}} p_{1}, \delta_{1}\right), \cdots$, $\left(2^{m_{0}} p_{k}, \delta_{1}\right)$ and the elements directly below $\left(2^{n_{0}}, \delta_{2}\right)$ in $\mathcal{D}^{1}\left(\beta_{2}, \delta_{2}\right)$ are $\left(2^{n_{0}} q_{1}, \delta_{2}\right), \cdots,\left(2^{n_{0}} q_{s}, \delta_{2}\right)$. Since $f$ must take the elements directly below $\left(2^{m_{0}}, \delta_{1}\right)$ to the elements directly below $\left(2^{n_{0}}, \delta_{2}\right)$, it follows that $k=s$.

The elements directly above $\left(b_{0}, \delta_{1}\right)$ in $\mathcal{D}^{1}\left(\beta_{1}, \delta_{1}\right)$ are listed as

$$
\begin{aligned}
& \left(2^{m_{0}} p_{1}^{m_{1}-1} \prod_{i \neq 1} p_{i}^{m_{i}}, \delta_{1}\right),\left(2^{m_{0}} p_{2}^{m_{2}-1} \prod_{i \neq 2} p_{i}^{m_{i}}, \delta_{1}\right), \cdots, \\
& \left(2^{m_{0}} p_{k}^{m_{k}-1} \prod_{i \neq k} p_{i}^{m_{i}}, \delta_{1}\right)
\end{aligned}
$$

Similarly the elements directly above $\left(c_{0}, \delta_{2}\right)$ are

$$
\begin{aligned}
& \left(2^{n_{0}} q_{1}^{n_{1}-1} \prod_{i \neq 1} q_{i}^{n_{i}}, \delta_{2}\right),\left(2^{n_{0}} q_{2}^{n_{2}-1} \prod_{i \neq 2} q_{i}^{n_{i}}, \delta_{2}\right), \cdots, \\
& \left(2^{n_{0}} q_{k}^{n_{k}-1} \prod_{i \neq k} q_{i}^{n_{i}}, \delta_{2}\right) .
\end{aligned}
$$

By reordering we may assume

$$
f\left(2^{m_{0}} p_{j}^{m_{j}-1} \prod_{i \neq j} p_{i}^{m_{i}}, \delta_{1}\right)=\left(2^{n_{0}} q_{j}^{n_{j}-1} \prod_{i \neq j} q_{i}^{n_{i}}, \delta_{2}\right)
$$

for $1 \leq j \leq k$. The number of elements in $\mathcal{D}^{1}\left(\beta_{1}, \delta_{1}\right)$ is $\prod_{i=1}^{k}\left(m_{i}+1\right)$, and this equals the number of elements in $\mathcal{D}^{1}\left(\beta_{2}, \delta_{2}\right)$ which is $\prod_{i=1}^{k}\left(n_{i}+1\right)$. Let

$$
\begin{aligned}
\mathcal{D}_{j}^{1}\left(\beta_{1}, \delta_{1}\right)= & \left\{\left(b, \delta_{1}\right) \in \mathcal{D}^{1}\left(\beta_{1}, \delta_{1}\right):\right. \\
& \left.\left(2^{m_{0}} p_{j}^{m_{j}-1} \prod_{i \neq j} p_{i}^{m_{i}}, \delta_{1}\right) \leq\left(b, \delta_{1}\right)\right\} .
\end{aligned}
$$

Similarly let

$$
\begin{aligned}
\mathcal{D}_{j}^{1}\left(\beta_{2}, \delta_{2}\right)= & \left\{\left(c, \delta_{2}\right) \in \mathcal{D}^{1}\left(\beta_{2}, \delta_{2}\right):\right. \\
& \left.\left(2^{n_{0}} q_{j}^{n_{j}-1} \prod_{i \neq j} q_{i}^{n_{i}}, \delta_{2}\right) \leq\left(c, \delta_{2}\right)\right\} .
\end{aligned}
$$

It follows that $f\left(\mathcal{D}_{j}^{1}\left(\beta_{1}, \delta_{1}\right)\right)=\mathcal{D}_{j}^{1}\left(\beta_{2}, \delta_{2}\right)$. Thus the number of elements in $\mathcal{D}_{j}^{1}\left(\beta_{1}, \delta_{1}\right)$ which is

$m_{j} \prod_{i \neq j}\left(m_{i}+1\right)$ is equal to $n_{j} \prod_{i \neq j}\left(n_{i}+1\right)$ the number of elements in $\mathcal{D}_{j}^{1}\left(\beta_{2}, \delta_{2}\right)$. Using the equations $\prod_{i \neq j}^{k}\left(m_{i}+1\right)=\prod_{i=1}^{k}\left(n_{i}+1\right)$ and

$$
m_{j} \prod_{i \neq j}\left(m_{i}+1\right)=n_{j} \prod_{i \neq j}\left(n_{i}+1\right),
$$

we obtain

$$
\frac{m_{j}}{n_{j}}=\frac{\prod_{i \neq j}\left(n_{i}+1\right)}{\prod_{i \neq j}\left(m_{i}+1\right)}=\frac{m_{j}+1}{n_{j}+1},
$$

and this implies that $m_{j}=n_{j}$ for $1 \leq j \leq k$.

We now suppose that $\left(2^{m_{0}} b_{0}, \delta_{1}\right)$ and $\left(2^{n_{0}} c_{0}, \delta_{2}\right)$ are the minimal elements in $\mathcal{D}^{1}\left(\beta_{1}, \delta_{1}\right)$ and $\mathcal{D}^{1}\left(\beta_{2}, \delta_{2}\right)$ respectively, and $b_{0}=\prod_{i=1}^{k} p_{i}^{m_{i}}$ and $c_{0}=\prod_{i=1}^{k} q_{i}^{m_{i}}$ are the prime decompositions. If $\left(b, \delta_{1}\right) \in \mathcal{D}^{1}\left(\beta_{1}, \delta_{1}\right)$, then 
$b=2^{m_{0}} \prod_{i=1}^{k} p_{i}^{s_{i}}$ where $0 \leq s_{i} \leq m_{i}$. Define

$f: \mathcal{D}^{1}\left(\beta_{1}, \delta_{1}\right) \rightarrow \mathcal{D}^{1}\left(\beta_{2}, \delta_{2}\right)$ by $f\left(b, \delta_{1}\right)=\left(2^{n_{0}} \prod_{i=1}^{k} q_{i}^{s_{i}}, \delta_{2}\right)$.

It is not hard to check that $f$ is an isomorphism. The proof in cases (2) - (4) is similar.

We now assume that $\delta_{1}$ is odd and $\delta_{2}$ is even with $n_{0}=0$. Since the argument is similar to the previous case, we will sketch the proof. The elements directly below $\left(2^{m_{0}}, \delta_{1}\right)$ in $\mathcal{D}^{1}\left(\beta_{1}, \delta_{1}\right)$ are $\left(2^{m_{0}} p_{1}, \delta_{1}\right), \cdots,\left(2^{m_{0}} p_{k}, \delta_{1}\right)$ and the elements directly below $\left(1, \delta_{2} / 2\right)$ in $\mathcal{D}^{1}\left(\beta_{2}, \delta_{2}\right)$ are $\left(q_{1}, \delta_{2} / 2\right), \cdots,\left(q_{s}, \delta_{2} / 2\right),\left(1, \delta_{2}\right)$. It follows that $k=s+$ 1. The elements in $\mathcal{D}^{1}\left(\beta_{1}, \delta_{1}\right)$ directly above $\left(2^{m_{0}} b_{0}, \delta_{1}\right)$ are

$$
\begin{aligned}
& \left(2^{m_{0}} p_{1}^{m_{1}-1} \prod_{i \neq 1} p_{i}^{m_{i}}, \delta_{1}\right),\left(2^{m_{0}} p_{2}^{m_{2}-1} \prod_{i \neq 2} p_{i}^{m_{i}}, \delta_{1}\right), \cdots, \\
& \left(2^{m_{0}} p_{k}^{m_{k}-1} \prod_{i \neq k} p_{i}^{m_{i}}, \delta_{1}\right),
\end{aligned}
$$

and the elements directly above $\left(c_{0}, \delta_{2}\right)$ are

$$
\begin{aligned}
& \left(q_{1}^{n_{1}-1} \prod_{i \neq 1} q_{i}^{n_{i}}, \delta_{2}\right),\left(q_{2}^{n_{2}-1} \prod_{i \neq 2} q_{i}^{n_{i}}, \delta_{2}\right), \cdots, \\
& \left(q_{k-1}^{n_{k-1}-1} \prod_{i \neq k-1} q_{i}^{n_{i}}, \delta_{2}\right),
\end{aligned}
$$

$\left(c_{0}, \delta_{2} / 2\right)$. By relabeling we may assume that

$$
f\left(2^{m_{0}} p_{j}^{m_{j}-1} \prod_{i \neq j} p_{i}^{m_{i}}, \delta_{1}\right)=\left(q_{j}^{n_{j}-1} \prod_{i \neq j} q_{i}^{n_{i}}, \delta_{2}\right)
$$

for $1 \leq j \leq k-1$ and

$$
f\left(2^{m_{0}} p_{k}^{m_{k}-1} \prod_{i \neq k} p_{i}^{m_{i}}, \delta_{1}\right)=\left(c_{0}, \delta_{2} / 2\right) .
$$

Now $\prod_{i=1}^{k}\left(m_{i}+1\right)=2 \prod_{i=1}^{k-1}\left(n_{i}+1\right)$, and for $j \leq k-1$ we have $m_{j} \prod_{i \neq j}\left(m_{i}+1\right)=2 n_{j} \prod_{i \neq j}\left(n_{i}+1\right)$.

Using these two equations we obtain $m_{j}=n_{j}$ for $1 \leq j \leq k-1$. The number of elements greater than or equal to $\left(2^{m_{0}} p_{k}^{m_{k}-1} \prod_{i \neq k} p_{i}^{m_{i}}, \delta_{1}\right)$ and $\left(c_{0}, \delta_{2} / 2\right)$ is

$m_{k} \prod_{i \neq k}\left(m_{i}+1\right)$ and $\prod_{i=1}^{k-1}\left(n_{i}+1\right)$ respectively. Since these two numbers must be equal, it follows that $m_{k}=1$.

For the converse suppose that $b_{0}=\prod_{i=1}^{k} p_{i}^{m_{i}}$ where $m_{k}=1$ and $c_{0}=\prod_{i=1}^{k-1} q_{i}^{m_{i}}$. If $\left(b, \delta_{1}\right)$ is any element in $\mathcal{D}^{1}\left(\beta_{1}, \delta_{1}\right)$, then $b=2^{m_{0}} \prod_{i=1}^{k} p_{i}^{s_{i}}$ where $0 \leq s_{i} \leq m_{i}$, and $s_{k}=0$ or 1 . Let $f\left(b, \delta_{1}\right)=\left(\prod_{i=1}^{k-1} q_{i}^{s_{1}}, \delta_{2} / 2\right)$ if $s_{k}=0$, and $\left(\prod_{i=1}^{k-1} q_{i}^{s_{1}}, \delta_{2}\right)$ if $s_{k}=1$. It follows that $f$ is an isomorphism.

For a pair of positive integers $\beta$ and $\delta$, let $\mathcal{L}^{2}(\beta, \delta)$ denote the equivalence classes of those actions whose quotient type is $\mathcal{O}_{2}(\beta, \delta)$. Let

$$
\begin{aligned}
& \mathcal{D}^{2}(\beta, \delta)=\left\{(b, d) \in \mathbb{Z}^{+} \times \mathbb{Z}^{+}: \text {g.c.d }\{b, d\}=1,\right. \\
& \left.b \text { divides } \beta, \frac{\beta}{b}=0(\bmod 2), \text { and } d=\delta \text { or } 2 d=\delta\right\} .
\end{aligned}
$$

It follows that $\mathcal{D}^{2}(\beta, \delta)$ is a partially ordered set under the ordering $\left(b_{2}, d_{2}\right) \geq\left(b_{1}, d_{1}\right)$ if $b_{2} \mid b_{1}, \frac{b_{1}}{b_{2}}=1(\bmod$ 2), and $d_{2} \mid d_{1}$.

The proof of the following theorem is similar to that of Theorem 12.

Theorem 15. For each pair of positive integers $\beta$ and $\delta$, the poset $\mathcal{L}^{2}(\beta, \delta)$ is isomorphic to the poset $\mathcal{D}^{2}(\beta, \delta)$.

We will now consider the structure of the partially ordered set $\mathcal{D}^{2}(\beta, \delta)$. Write $\beta=2^{m} b_{0} b_{1}$ where $b_{0}$ and $b_{1}$ are both odd and $b_{0}$ is the largest odd divisor of $\beta$ which is relatively prime to $\delta$.

Theorem 16. For each pair of positive integers $\beta$ and $\delta$, the poset $\mathcal{D}^{2}(\beta, \delta)$ is a disjoint union of lattices given by

$$
\begin{aligned}
& \mathcal{D}^{2}(\beta, \delta) \\
& = \begin{cases}\cup_{j=1}^{m} \mathcal{D}^{1}\left(2^{m-j} b_{0}, \delta\right) & \text { if } \delta \neq 0(\bmod 2) \\
\mathcal{D}^{1}\left(b_{0}, \delta\right) & \text { if } \delta=0(\bmod 4) \\
\cup_{j=1}^{m-1} \mathcal{D}^{1}\left(2^{m-j} b_{0}, \delta / 2\right) \cup \mathcal{D}^{1}\left(b_{0}, \delta\right) & \text { if } \frac{\delta}{2} \neq 0(\bmod 2)\end{cases}
\end{aligned}
$$

Proof. We first assume that $\delta$ is odd. Note that for $1 \leq j \leq m$, we have $\left(2^{m-j} b_{0}, \delta\right) \in \mathcal{D}^{2}(\beta, \delta)$. It suffices to show that if $\left(2^{m-j} b_{0}, \delta\right) \geq(b, d)$, then $\left(2^{m-j} b_{0}, \delta\right)=$ $(b, d)$ and hence $\left(2^{m-j} b_{0}, \delta\right)$ is a minimal element, and if $(b, d) \in \mathcal{D}^{2}(\beta, \delta)$, then $(b, d) \geq\left(2^{m-j} b_{0}, \delta\right)$ for some unique $j$ where $1 \leq j \leq m$. Suppose $\left(2^{m-j} b_{0}, \delta\right) \geq$ $(b, d)$, and thus $2^{m-j} b_{0}$ divides $b, \delta$ divides $d$, and

$\frac{b}{2^{m-j} b_{0}}$ is odd. Now $d$ divides $\delta$, which implies $d=\delta$. Since $b$ divides $\beta=2^{m} b_{0} b_{1}$ and g.c.d $\{b, \delta\}=1$, it follows that $b$ must divide $2^{m} b_{0}$. Write $b=2^{w} b^{\prime}$ where $b^{\prime}$ is odd. Now $\frac{2^{w} b^{\prime}}{2^{m-j} b_{0}}$ being odd implies $w=m-j$ and $b_{0}$ divides $b^{\prime}$. Note that $b=2^{m-j} b^{\prime}$ divides $2^{m} b_{0}$. Thus $b^{\prime}$ divides $b_{0}$ showing that $b^{\prime}=b_{0}$, and therefore $b=2^{m-j} b_{0}$. Let $(b, d) \in \mathcal{D}^{2}(\beta, \delta)$. Since $\delta$ is odd, it follows that $d=\delta$. As above we have that $b$ divides $2^{m} b_{0}$. Furthermore, $\frac{2^{m} b_{0}}{b}$ must be even. We may write $b=2^{r} b^{\prime}$ where $0 \leq r \leq m-1, b^{\prime}$ is odd, and $b^{\prime}$ divides $b_{0}$. Therefore $(b, d) \geq\left(2^{r} b_{0}, \delta\right)$.

We now assume that $\delta$ is even and we write $\delta=2^{n} \delta^{\prime}$ where $\delta^{\prime}$ is odd. There are two cases to consider: $n \geq 2$ and $n=1$. Suppose first that $n \geq 2$. Now $\left(b_{0}, \delta\right) \in$ $\mathcal{D}^{2}(\beta, \delta)$. We will show that $\left(b_{0}, \delta\right)$ is the minimal element in $\mathcal{D}^{2}(\beta, \delta)$. Suppose that $(b, d) \in \mathcal{D}^{2}(\beta, \delta)$ and $\left(b_{0}, \delta\right) \geq(b, d)$. In this case $d=\delta$. Also since $\frac{b}{b_{0}}$ 
and $b_{0}$ are both odd, it follows that $b$ is odd. Since g.c. $\{b, \delta\}=1$ and $b$ is odd, it follows that $b$ divides $b_{0}$. Thus $b=b_{0}$ showing that $\left(b_{0}, \delta\right)$ is the minimal element. Now let $(b, d) \in \mathcal{D}^{2}(\beta, \delta)$. Recall that $d=\delta$ or $2 d=\delta$. If $d=\delta$, then since $b$ and $d$ are relatively prime, $b$ must be odd. Furthermore, $b$ must divide $b_{0}$, and thus $(b, d) \geq\left(b_{0}, \delta\right)$. If $2 d=\delta$, then $d=\frac{\delta}{2}=2^{n-1} \delta^{\prime}$ and $d$ is even since $n \geq 2$. Now $b$ and $d=2^{n-1} \delta^{\prime}$ are relatively prime, which implies that $b$ is odd. Again we have that $b$ must divide $b_{0}$, so that $(b, d) \geq\left(b_{0}, \delta\right)$. This shows that $\left(b_{0}, \delta\right)$ is the minimal element and $\mathcal{D}^{2}(\beta, \delta)=\mathcal{D}^{1}\left(b_{0}, \delta\right)$. We now consider the case when $n=1$. Note that $\left(2^{m-j} b_{0}, \delta / 2\right) \in \mathcal{D}^{2}(\beta, \delta)$ for $1 \leq j \leq m$ -1 and $\left(b_{0}, \delta\right) \in \mathcal{D}^{2}(\beta, \delta)$. We need to show that these are the minimal elements in $\mathcal{D}^{2}(\beta, \delta)$, and if $(b, d) \in$ $\mathcal{D}^{2}(\beta, \delta)$ then either $(b, d) \geq\left(2^{m-j} b_{0}, \delta / 2\right)$ for some unique $j$ or $(b, d) \geq\left(b_{0}, \delta\right)$. The proof to this is similar to case 1 .

Remark 17. Note that by Theorem 7 each $\cup_{j=1}^{m} \mathcal{D}^{1}\left(2^{m-j} b_{0}, \delta\right)$ and $\cup_{j=1}^{m-1} \mathcal{D}^{1}\left(2^{m-j} b_{0}, \delta / 2\right)$ is a disjoint union of isomorphic lattices.

For a pair of positive integers $\beta$ and $\delta$ with $\beta>\delta$ and $\beta+\delta$ even, let $\mathcal{L}^{3}(\beta, \delta)$ denote the equivalence classes of those actions whose quotient type is $\mathcal{O}_{3}(\beta, \delta)$. Let

$$
\begin{aligned}
\mathcal{D}^{3}(\beta, \delta)= & \left\{(b, d) \in \mathbb{Z}^{+} \times \mathbb{Z}^{+}: d=\frac{\beta-\delta}{2},\right. \\
& \text { g.c.d } \left.\{b, d\}=1, b \text { divides } \frac{\beta+\delta}{2}\right\} .
\end{aligned}
$$

It follows that $\mathcal{D}^{3}(\beta, \delta)$ is a partially ordered set under the ordering $\left(b_{2}, d\right) \geq\left(b_{1}, d\right)$ if $b_{2} \mid b_{1}$ and $\frac{b_{1}}{b_{2}}=1(\bmod$ 2).

Theorem 18. For each pair of positive integers $\beta$ and $\delta$ with $\beta>\delta$ and $\beta+\delta$ even, the poset $\mathcal{L}^{3}(\beta, \delta)$ is isomorphic to the poset $\mathcal{D}^{3}(\beta, \delta)$.

Proof. Let $(b, d) \in \mathcal{D}^{3}(\beta, \delta)$ and let $m=\frac{\beta+\delta}{2 b}$. Observe that $b m+d=\beta$ and $b m-d=\delta$. There exists a standard $\mathbb{Z}_{2 m}$-action $\varphi: \mathbb{Z}_{2 m} \rightarrow \operatorname{Diff}(M(b, d))$ such that

$$
M(b, d) / \varphi=\mathcal{O}_{3}(b m+d, b m-d)=\mathcal{O}_{3}(\beta, \delta)
$$

where $\varphi=\alpha_{3}$ if $m$ is odd and $\varphi=\beta_{3}$ if $m$ is even. Define $f: \mathcal{D}^{3}(\beta, \delta) \rightarrow \mathcal{L}^{3}(\beta, \delta)$ by $f(b, d)=[\varphi] \in \mathcal{L}^{3}(\beta, \delta)$.

Suppose that $\left(b_{2}, d\right) \geq\left(b_{1}, d\right)$. Let $\frac{\beta+\delta}{2 b_{1}}=m_{1}$ and $\frac{\beta+\delta}{2 b_{2}}=m_{2}$ and let $\varphi_{1}: \mathbb{Z}_{2 m_{1}} \rightarrow \operatorname{Diff}\left(M\left(b_{1}, d\right)\right)$ and $\varphi_{2}: \mathbb{Z}_{2 m_{2}} \rightarrow \operatorname{Diff}\left(M\left(b_{2}, d\right)\right)$ be the standard actions. It follows that $m_{2}=m_{1}\left(\frac{b_{1}}{b_{2}}\right)$, and therefore $\mathbb{Z}_{2 m_{1}}$ is a subgroup of $\mathbb{Z}_{2 m_{2}}$. Furthermore, $\left(\varphi_{2}(1)\right)^{\frac{b_{1}}{b_{2}}}=\varphi_{1}(1)$, which implies that $\left[\varphi_{2}\right] \geq\left[\varphi_{1}\right]$ and thus $f$ is order preserving. The proof that $f$ is one-to-one and onto is similar to that in Theorem 12.

We will now consider the structure of the partially ordered set $\mathcal{D}^{3}(\beta, \delta)$. Write $\frac{\beta+\delta}{2}=2^{m} \gamma$ where $\gamma$ is odd. Let $b_{0}$ be the largest positive odd divisor of $\gamma$ which is relatively prime to $d=\frac{\beta-\delta}{2}$.

Theorem 19. For each pair of positive integers $\beta$ and $\delta$ with $\beta>\delta, \beta+\delta$ even, and $d=\frac{\beta-\delta}{2}$, the poset $\mathcal{D}^{3}(\beta, \delta)$ is a disjoint union of isomorphic lattices given by

$$
\mathcal{D}^{3}(\beta, \delta)= \begin{cases}\cup_{j=0}^{m} \mathcal{D}^{1}\left(2^{m-j} b_{0}, d\right) & \text { if } \beta-\delta \neq 0(\bmod 4) \\ \mathcal{D}_{*}^{1}\left(b_{0}, d\right) & \text { if } \beta-\delta=0(\bmod 4)\end{cases}
$$

Proof. Suppose first that $\beta-\delta \neq 0(\bmod 4)$ (equivalently $d$ is odd). Note that $2^{j} b_{0}$ divides $\frac{\beta+\delta}{2}$ for $0 \leq j \leq m$, and since $d$ is odd we have $\operatorname{g.c.d}\left\{2^{j} b_{0}, d\right\}=1$. It follows that $\left(2^{j} b_{0}, d\right) \in \mathcal{D}^{3}(\beta, \delta)$ and $\left(2^{j} b_{0}, d\right)$ is a minimal element of $\mathcal{D}^{1}\left(2^{j} b_{0}, d\right)$. Let $(b, d) \in \mathcal{D}^{3}(\beta, \delta)$. Write $b=2^{k} b^{\prime}$ where $b^{\prime}$ is odd. Since $b$ divides $\frac{\beta+\delta}{2}=2^{m} \gamma$, it follows that $0 \leq k \leq m$ and $b^{\prime}$ divides $\gamma$. Furthermore, $b^{\prime}$ and $d$ are relatively prime. Since $b_{0}$ is the largest positive odd divisor of $\gamma$ which is relatively prime to $d$, it follows that $b^{\prime}$ divides $b_{0}$. Hence $(b, d) \in \mathcal{D}^{1}\left(2^{k} b_{0}, d\right)$ for a unique $k$. Now suppose $(b, d) \in \mathcal{D}^{1}\left(2^{k} b_{0}, d\right)$ for some $k$. By assumption $d=\frac{\beta-\delta}{2}$. Since $b$ divides $2^{k} b_{0}$ and $2^{k} b_{0}$ divides $2^{m} \gamma=\frac{\beta+\delta}{2}$, it follows that $b$ divides $\frac{\beta+\delta}{2}$, and hence $(b, d) \in \mathcal{D}^{3}(\beta, \delta)$.

The proof for $\beta-\delta=0(\bmod 4)$ is similar.

Corollary 20.

$$
\mathcal{L}^{3}(\beta, \delta) \simeq \begin{cases}\cup_{j=0}^{m} \mathcal{D}^{1}\left(2^{m-j} b_{0}, d\right) & \text { if } \beta-\delta \neq 0(\bmod 4) \\ \mathcal{D}_{*}^{1}\left(b_{0}, d\right) & \text { if } \beta-\delta=0(\bmod 4)\end{cases}
$$

For each pair of positive integers $\beta$ and $\delta$, let $\mathcal{L}^{4}(\beta, \delta)$ denote the set of equivalence classes of actions 
on prisim manifolds whose quotient space is $\mathcal{O}_{4}(\beta, \delta)$.

Theorem 21. $\mathcal{L}^{4}(\beta, \delta) \simeq \mathcal{D}_{*}^{1}(\beta, \delta)$

Proof. If $[\varphi] \in \mathcal{L}^{4}(\beta, \delta)$, then $\varphi$ is equivalent to $\alpha_{5}: \operatorname{Dih}\left(\mathbb{Z}_{2 n+1}\right) \rightarrow \operatorname{Diff}(M(b, d))$ for some integers $n$, $b$, and $d$ where $\beta=b(2 n+1)$ and $\delta=d$. Since $(b, d) \in$ $\mathcal{D}_{*}^{1}(\beta, \delta)$, define a function $f: \mathcal{L}^{4}(\beta, \delta) \rightarrow \mathcal{D}_{*}^{1}(\beta, \delta)$ by $f([\varphi])=(b, d)$. It follows easily that $f$ is an order preserving surjection.

For each pair of positive integers $\beta$ and $\delta$, let $\mathcal{L}^{5}(\beta, \delta)$ denote the set of equivalence classes of actions on prisim manifolds whose quotient space is $\mathcal{O}_{5}(\beta, \delta)$. We now consider the structure of the partially ordered set $\mathcal{L}^{5}(\beta, \delta)$. Write $\beta=2^{m} b_{0} b_{1}$ where $b_{1}$ is odd and $b_{0}$ is the largest odd divisor of $\beta$ that is relatively prime to $\delta$.

Theorem 22.

$\mathcal{L}^{5}(\beta, \delta)$

$$
\simeq \begin{cases}\cup_{j=0}^{m} \mathcal{D}^{1}\left(2^{j} b_{0}, \delta\right) & \text { if } \delta \neq 0(\bmod 2) \text { and } m \geq 1 \\ \mathcal{D}_{*}^{1}\left(b_{0}, \delta\right) & \text { if } \delta=0(\bmod 4) \text { and } m \geq 1 \\ \mathcal{D}^{1}\left(2^{m} b_{0}, \delta / 2\right) \cup \mathcal{D}_{*}^{1}(\beta, \delta) & \text { if } \frac{\delta}{2} \neq 0(\bmod 2) \text { and } m \geq 1 \\ \mathcal{D}^{1}\left(b_{0}, \delta\right) & \text { if } m=1\end{cases}
$$

Proof. Suppose that $\delta \neq 0(\bmod 2)$. Let $[\varphi] \in \mathcal{L}^{5}(\beta, \delta)$. We have a covering $M(b, d) \rightarrow \mathcal{O}_{5}(\beta, \delta)=M(b, d) / \varphi$ for some positive integers $b$ and $d$. Now $\varphi$ is equivalent to either of the standard actions $\alpha_{5}, \gamma_{5}$ or $\delta_{5}$. The action $\beta_{5}$ is impossible since $\delta$ is odd. We will define a function $f: \mathcal{L}^{5}(\beta, \delta) \rightarrow \cup_{j=0}^{m} \mathcal{D}^{1}\left(2^{j} b_{0}, \delta\right)$ as follows: if $\varphi$ is equivalent to $\alpha_{5}$, then $b(2 n+1)=2^{m} b_{0} b_{1}$ for some $n$ and $d=\delta$. Since $b$ and $d$ are relatively prime and $b_{0}$ is the largest odd divisor of $\beta$ that is relatively prime to $\delta$, it follows that $b$ divides $2^{m} b_{0}$. Thus $(b, d) \in \mathcal{D}^{1}\left(2^{m} b_{0}, \delta\right)$ and we let

$$
f([\varphi])=(b, d) \in \mathcal{D}^{1}\left(2^{m} b_{0}, \delta\right) .
$$

If $\varphi$ is equivalent to $\gamma_{5}$, then $b(4 n+2)=2^{m} b_{0} b_{1}$, and this implies that $b$ must divide $2^{m-1} b_{0}$. Thus $(b, d) \in$ $\mathcal{D}^{1}\left(2^{m-1} b_{0}, \delta\right)$ and we define

$$
f([\varphi])=(b, d) \in \mathcal{D}^{1}\left(2^{m-1} b_{0}, \delta\right) .
$$

If $\varphi$ is equivalent to $\delta_{5}$, then $4 n b=2^{m} b_{0} b_{1}$ for some $n$. Write $n=2^{k} n_{0}$ where $n_{0}$ is odd. This implies that $b$ divides $2^{m-k-2} b_{0}$ where $0 \leq k \leq m-2$. This shows that $(b, d) \in \mathcal{D}^{1}\left(2^{m-k-2} b_{0}, \delta\right)$ and we define

$$
f([\varphi])=(b, d) \in \mathcal{D}^{1}\left(2^{m-k-2} b_{0}, \delta\right) .
$$

We now show that $f$ is an order preserving bijection. Note that there do not exist integers $n, n^{\prime}, b$, and $b^{\prime}$, such that $b^{\prime}\left(2 n^{\prime}+1\right)=2 b(2 n+1)$, or $b^{\prime}\left(2 n^{\prime}+1\right)=4 n b$, or $2 b^{\prime}\left(2 n^{\prime}+1\right)=4 n b$, if either $b$ divides $b^{\prime}$ or $b^{\prime}$ divides $b$ with odd quotient. This implies that $f$ is one-to- one. Furthermore if $\left[\varphi_{2}\right] \geq\left[\varphi_{1}\right]$, then $\varphi_{2}$ and $\varphi_{1}$ are both equivalent to either $\alpha_{5}, \gamma_{5}$ or $\delta_{5}$. From this it can be shown that $f\left(\left[\varphi_{2}\right]\right) \geq f\left(\left[\varphi_{1}\right]\right)$. To show $f$ is onto, suppose $(b, d) \in \mathcal{D}^{1}\left(2^{j} b_{0}, \delta\right)$ for $0 \leq j \leq m$. Let

$\frac{2^{j} b_{0}}{b}=2 n^{\prime}+1$ for some positive integer $n^{\prime}$. If $m=j$, then $\beta=2^{m} b_{0} b_{1}=b\left(2 n^{\prime}+1\right) b_{1}$, and since $b_{1}$ is odd we may write $\beta=b(2 n+1)$. Hence there is an action

$$
\alpha_{5}: \operatorname{Dih}\left(\mathbb{Z}_{2 n+1}\right) \rightarrow \operatorname{Diff}(M(b, d))
$$

such that $M(b, d) / \alpha_{5}=\mathcal{O}_{5}(\beta, \delta)$, and thus $f\left(\left[\alpha_{5}\right]\right)=$ $(b, d)$. Similarly, if $j=m-1$ or if $j<m-1$, we obtain actions $\gamma_{5}$ and $\delta_{5}$-actions respectively. This shows that $f$ is onto.

If $\delta=0(\bmod 4)$, then if $m=1$ there exist only $\gamma_{5}$ actions, and if $m>1$ there exist only $\delta_{5}$-actions. For $\frac{\delta}{2} \neq 0 \quad(\bmod 2)$, if $m=1$ there exist only $\beta_{5}$ and $\gamma_{5}-$ actions, and if $m>1$ there exist only $\beta_{5}$ and $\delta_{5}$-actions. If $\beta$ is odd and $\delta$ is even, then there exist only $\alpha_{5}$ and $\beta_{5}$-actions. The proof in all these cases is similar to the above.

For each pair of positive integers $\beta$ and $\delta$ with $\beta>\delta$ and $\beta+\delta$ even, let $\mathcal{L}^{6}(\beta, \delta)$ denote the set of equivalence classes of actions on prisim manifolds whose quotient space is $\mathcal{O}_{6}(\beta, \delta)$.

Theorem 23. For each pair of positive integers $\beta$ and $\delta$, the poset $\mathcal{L}^{6}(\beta, \delta)$ is isomorphic to the poset $\mathcal{D}^{3}(\beta, \delta)$.

Proof. Let $[\varphi] \in \mathcal{L}^{6}(\beta, \delta)+1$. Now $\varphi$ is a $\operatorname{Dih}\left(\mathbb{Z}_{n}\right)$ action on a prisim manifold $M(b, d)$ and is equivalent to $\alpha_{6}$ if $n$ is even or $\beta_{6}$ if $n$ is odd. Furthermore,

$$
M(b, d) / \varphi=\mathcal{O}_{6}(b n+d, b n-d),
$$

and therefore $\beta=b n+d$ and $\delta=b n-d$. It follows that $(b, d) \in \mathcal{D}^{3}(\beta, \delta)$. Define a function $f: \mathcal{L}^{6}(\beta, \delta) \rightarrow$ $\mathcal{D}^{3}(\beta, \delta)$ by $f([\varphi])=(b, d)$.

Let $(b, d) \in \mathcal{D}^{3}(\beta, \delta)$. Therefore $b n=\frac{\beta+\delta}{2}$ and $d=\frac{\beta-\delta}{2}$ for some $n \in \mathbb{Z}$. This implies $b n+d=\beta$ and $b n-d=\delta$. If $n$ is even there exists an $\alpha_{6}$-action, and if $n$ is odd there exists an $\beta_{6}$-action. Therefore $f$ is onto.

Let $f\left(\left[\varphi_{1}\right]\right)=\left(b_{1}, d_{1}\right)$ and $f\left(\left[\varphi_{2}\right]\right)=\left(b_{2}, d_{2}\right)$ and suppose $f\left(\left[\varphi_{1}\right]\right)=f\left(\left[\varphi_{2}\right]\right)$. Then $\left(b_{1}, d_{1}\right)=\left(b_{2}, d_{2}\right)$ and hence $b_{1}=b_{2}$ and $d_{1}=d_{2}$. We also have $b_{1} n_{1}+d_{1}=\beta$ and $b_{2} n_{2}+d_{2}=\beta$ so that $n_{1}=n_{2}$. Recall that $\varphi_{1}$ and $\varphi_{2}$ are equivalent to either an $\alpha_{6}$ or $\beta_{6}$-action. If $n_{1}=n_{2}$ is even, then both of them are equivalent to an $\alpha_{6}$-action, otherwise they are both equivalent to a $\beta_{6}$ - 
action. Hence $\varphi_{1}$ and $\varphi_{2}$ are equivalent showing $f$ is one-to-one.

If $\left[\varphi_{1}\right] \leq\left[\varphi_{2}\right]$, then there is a covering map

$$
v: M\left(b_{2}, d_{2}\right) \rightarrow M\left(b_{1}, d_{1}\right)
$$

where $d_{1}=d_{2}$. Hence, $(2 n+1) b_{2}=b_{1}$ for some $n \in \mathbb{Z}$ which shows $b_{2} \mid b_{1}$ is an odd number. Therefore, we conclude $\left(b_{1}, d_{1}\right) \leq\left(b_{2}, d_{2}\right)$ showing $f$ is order preserving.

For each pair of positive integers $\beta$ and $\delta$, let $\mathcal{L}^{7}(\beta, \delta)$ denote the set of equivalence classes of actions on prisim manifolds whose quotient space is $\mathcal{O}_{7}(\beta, \delta)$. The proof of the following theorem is similar to that of Theorem 23 .

Theorem 24. For each pair of positive integers $\beta$ and $\delta$, the poset $\mathcal{L}^{7}(\beta, \delta)$ is isomorphic to the poset $\mathcal{D}^{2}(\beta, \delta)$.

\section{Primitive Actions on Prism Manifolds}

Let $\varphi: G \rightarrow \operatorname{Diff}(M(b, d))$ be a $G$-action on a prism manifold $M(b, d)$ with orbifold covering map

$$
v_{\varphi}: M(b, d) \rightarrow M(b, d) / \varphi .
$$

We say that $\varphi$ is primitive if $G$ does not contain a nontrivial normal subgroup which acts freely on $M(b, d)$. Therefore for any nontrivial normal subgroup $H$ of $G$, if

$$
\varphi_{0}=\left.\varphi\right|_{H}: H \rightarrow \operatorname{Diff}(M(b, d)),
$$

then $M(b, d) / \varphi_{0}$ is not a manifold. In this section we determine when an action is primitive.

Theorem 25. Let $\theta: \mathbb{Z}_{m} \rightarrow \operatorname{Diff}(M(b, d))$ be a $\mathbb{Z}_{m}$ action on the prism manifold $M(b, d)$.

1) If $\theta$ is equivalent to $\alpha_{1}$, then $\theta$ is primitive if and only if for every prime divisor $p$ of $m, d=0 \quad(\bmod$ p).

2) If $\theta$ is equivalent to $\beta_{1}$, then $\theta$ is primitive if and only if $b$ is even and for every odd prime divisor $p$ of $m, d=0(\bmod p)$.

3) If $\theta$ is equivalent to $\alpha_{2}$ or $\beta_{3}$, then $\theta$ is primitive if and only if either $m=2^{n}$, or if $p$ is any odd prime divisor of $m$, then $d=0(\bmod p)$.

4) If $\theta$ is equivalent to $\alpha_{3}$, then $\theta$ is primitive if and only if either $m=2$, or if $p$ is any odd prime divisor of $m$, then $d=0(\bmod p)$.

Proof. We may suppose $\theta=\alpha_{1}$. Then $m=2 n+1$ and if $\mathbb{Z}_{l}$ is a subgroup of $\mathbb{Z}_{m}$ and $\theta_{0}=\left.\theta\right|_{\mathbb{Z}_{l}}: \mathbb{Z}_{l} \rightarrow$ $\operatorname{Diff}(M(b, d))$, then $M(b, d) \rightarrow \mathcal{O}_{1}(b l, d)=M(b, d) / \theta_{0}$. Furthermore $\mathcal{O}_{1}(b l, d)$ is a manifold if and only if g.c. $\{b l, d\}=1$. Assume that $\theta$ is primitive and let $p$ be a prime divisor of $m$. Consider the subgroup $\mathbb{Z}_{p}$. Since g.c.d $\{b, d\}=1$ and $\theta$ is primitive, it follows that g.c. $\{\{b p, d\}=$ g.c. $d\{p, d\}=p$. Thus $p$ divides $d$. Now suppose that every prime divisor of $m$ also divides $d$ and let $\mathbb{Z}_{l}$ be a subgroup of $\mathbb{Z}_{m}$. Let $p$ be a prime divisor of $l$. Since $l$ divides $m$, it follows that $p$ divides $m$. Hence by assumption g.c.d $\{b l, d\} \neq 1$, showing that $\theta$ is primitive.

For part 2), suppose that $\theta=\beta_{1}$. Then $m=2(2 n+1)$ and if $\mathbb{Z}_{l}$ is a subgroup of $\mathbb{Z}_{m}$ and $\theta_{0}=\left.\theta\right|_{\mathbb{Z}_{l}}: \mathbb{Z}_{l} \rightarrow$ $\operatorname{Diff}(M(b, d))$, then either $l=2 s+1$ and

$$
M(b, d) \rightarrow \mathcal{O}_{1}(b l, d)=M(b, d) / \theta_{0},
$$

or $l=2(2 s+1)$ and

$$
M(b, d) \rightarrow \mathcal{O}_{1}(b(2 s+1), 2 d)=M(b, d) / \theta_{0} .
$$

Furthermore $\mathcal{O}_{1}(b l, d)$ is a manifold if and only if g.c.d $\{b l, d\}=1$; and $\mathcal{O}_{1}(b(2 s+1), 2 d)$ is a manifold if and only if g.c.d $\{b(2 s+1), 2 d\}=1$.

Assume first that $\theta$ is primitive. If $p$ is an odd prime divisor of $m$, then the same argument used in the $\alpha_{1}$ case shows $p$ divides $d$. Now $\mathbb{Z}_{2}$ is a subgroup of $\mathbb{Z}_{m}$ and we have a covering

$$
M(b, d) \rightarrow \mathcal{O}_{1}(b, 2 d)=M(b, d) / \mathbb{Z}_{2} .
$$

Since $\theta$ is primitive, $\mathcal{O}_{1}(b, 2 d)$ is not a manifold, and since g.c.d $\{b, d\}=1$ it follows that 2 divides $b$. For the converse suppose that $b$ is even, and if $p$ is any odd prime divisor of $m$, then $d=0(\bmod p)$. Let $\mathbb{Z}_{l}$ be any subgroup of $\mathbb{Z}_{m}$. If $l$ is odd, the proof that $\theta$ is primitive is identical to the $\alpha_{1}$ case. If $l$ is even, then g.c.d $\{b(2 s+1), 2 d\} \neq 1$, showing that $\theta$ is primitive.

To prove part 3), suppose that $\theta$ is equivalent to $\alpha_{2}$. Therefore $m=2 s$ and $\alpha_{2}: \mathbb{Z}_{2 s} \rightarrow \operatorname{Diff}(M(b, d))$ is defined by $\alpha_{2}(1)([r u])=\left[r u e^{\frac{\pi i}{s}}\right]$ where $[r u]$ is any point in the Heegaard Klein bottle $K$ and 1 denotes a generator of $\mathbb{Z}_{2 s}$. If $\mathbb{Z}_{l}$ is any subgroup of $\mathbb{Z}_{2 s}$ and $\alpha=\left.\alpha_{2}\right|_{\mathbb{Z}_{l}}: \mathbb{Z}_{l} \rightarrow \operatorname{Diff}(M(b, d))$, then $\alpha(1)([r u])=\left[r u e^{\frac{2 \pi i}{l}}\right]$ where 1 denotes a generator of $\mathbb{Z}_{l}$.

We now suppose that $\alpha_{2}$ is primitive and $p$ be an odd prime divisor of $m=2 s$. Letting $l=p$, we obtain a covering $M(b, d) \rightarrow M(b, d) / \alpha=\mathcal{O}_{1}(b p, d)$. Since $\alpha_{2}$ is primitive $g . c . d\{b p, d\} \neq 1$, and since g.c. $\{b p, d\}=$ g.c. $d\{p, d\}$, it follows that $p$ divides $d$.

Suppose $m=2^{n}$. Then $l=2^{t}$ and $\alpha(1)([r u])=\left[r u e^{\frac{2 \pi i}{2^{t}}}\right]=\left[r u e^{\frac{\pi i}{2^{t-1}}}\right]$. Now $K / \alpha$ is a mirrored annulus, showing that $M(b, d) / \alpha$ is not a manifold, and thus $\alpha_{2}$ is primitive. Suppose now that $m \neq 2^{n}$, and if $p$ is an odd prime divisor of $m$, then $d=0(\bmod$ $p)$. Assume there is a covering $M(b, d) \rightarrow M(b, d) / \alpha=\mathcal{O}_{1}(b l, d)$ (Note that the quotient space cannot be $\mathcal{O}_{1}(b l, 2 d)$ by definition of $\alpha_{2}$ 
and $\beta_{1}$ ). It follows that $l$ is odd. Let $p$ be any odd prime divisor of $l$. It follows that $p$ divides $m$, and hence by assumption $p$ divides $d$. Thus g.c.d $\{b l, d\} \neq 1$, showing $\alpha_{2}$ is primitive.

We now suppose that $\theta=\beta_{3}$. In this case $m=4 s$ and $\beta_{3}: \mathbb{Z}_{4 s} \rightarrow \operatorname{Diff}(M(b, d))$ where

$\beta_{3}(1)([r u])=\left[\frac{1}{r} u e^{\frac{\pi i}{2 s}}\right]$. If $\mathbb{Z}_{l}$ is a subgroup of $\mathbb{Z}_{m}$, let $\beta=\left.\beta_{3}\right|_{\mathbb{Z}_{l}}: \mathbb{Z}_{l} \rightarrow \operatorname{Diff}(M(b, d))$. Assume first that $\theta$ is primitive. Suppose $p$ is an odd prime divisor of $m$ and consider the primitive subgroup $\mathbb{Z}_{p}$ of $\mathbb{Z}_{m}$. Here $l=p$, and since $\frac{m}{p}$ is even we have $\beta(1)([r u])=\left[r u e^{\frac{2 \pi i}{p}}\right]$.

Since $\beta$ is a primitive $\alpha_{1}$-action on $M(b, d)$, it follows by the above that $d=0(\bmod p)$. Now suppose $m=2^{n}$ and let $\mathbb{Z}_{l}$ be a subgroup of $\mathbb{Z}_{m}$. Then $l=2^{t}$ and $\beta(1)([r u])=\left[r u e^{\frac{\pi i}{2^{t-1}}}\right]$. In this case $\beta$ is an $\alpha_{2}-$ action and $M(b, d) / \beta$ is not a manifold.

Now suppose that every odd prime divisor of $\mathrm{m}$ also divides $d$. Let $\mathbb{Z}_{l}$ be a subgroup of $\mathbb{Z}_{m}$. If $l$ is odd, then $\frac{4 s}{l}$ is even and $\beta(1)([r u])=\left[r u e^{\frac{2 \pi i}{l}}\right]$ which is an $\alpha_{1}$ action. The result follows by the above that $\beta$ is primitive, and thus $\theta$ is primitive. Now suppose $l$ is even. If $\frac{m}{l}$ is even then $\beta(1)([r u])=\left[r u e^{\frac{2 \pi i}{l}}\right]=\left[r u e^{\frac{\pi i}{l^{\prime}}}\right]$, which is an $\alpha_{2}$-action; and if $\frac{m}{l}$ is odd then

$\beta(1)([r u])=\left[\frac{1}{r} u e^{\frac{2 \pi i}{l}}\right]=\left[\frac{1}{r} u e^{\frac{\pi i}{l^{\prime}}}\right]$, which is a $\beta_{3}$-action.

In either case the quotient is not a manifold. Hence $\theta$ is primitive.

We now prove part 4) and suppose $\theta=\alpha_{3}$. Therefore $m=2(2 n+1)$ and $\alpha_{3}: \mathbb{Z}_{2(2 n+1)} \rightarrow \operatorname{Diff}(M(b, d))$ where $\alpha_{3}(1)([r u])=\left[\frac{1}{r} u e^{\frac{2 \pi i}{2 n+1}}\right]$. If $m=2$, then $K / \alpha_{3}$ is a mirrored Möbius band and the action is primitive. So suppose that $m \neq 2$. Assume first that $\alpha_{3}$ is primitive and let $p$ be an odd divisor of $m$, and hence $p$ divides $2 n+1$. Consider the subgroup $\mathbb{Z}_{p}$ and let

$$
\alpha=\left.\alpha_{3}\right|_{\mathbb{Z}_{p}}: \mathbb{Z}_{p} \rightarrow \operatorname{Diff}(M(b, d)) .
$$

Then $\alpha(1)([r u])=\left[r u e^{\frac{4 \pi i}{p}}\right]$, and we have a covering $M(b, d) \rightarrow M(b, d) / \alpha=\mathcal{O}_{1}(b p, d)$. Again as above since $\alpha_{3}$ is primitive, $p$ must divide $d$.
We now suppose that for each odd prime divisor $p$ of $m, d=0(\bmod p)$. Let $\mathbb{Z}_{l}$ be a subgroup of $\mathbb{Z}_{m}$. If $l$ is odd we obtain a covering

$M(b, d) \rightarrow M(b, d) / \mathbb{Z}_{l}=\mathcal{O}_{1}(b l, d)$, and as above if $p$ is a prime divisor of $l$ we obtain $g . c . d\{b l, d\} \neq 1$. Thus the action is primitive. If $l$ is even then $l=2(2 s+1)$, and if $\alpha=\left.\alpha_{3}\right|_{\mathbb{Z}_{l}}$ then $\alpha(1)([r u])=\left[\frac{1}{r} u e^{\frac{2 \pi i}{2 s+1}}\right]$. In this case $M(b, d) / \mathbb{Z}_{l}=\mathcal{O}_{3}(b l, d)$, which is not a manifold showing that the action is primitive.

Proposition 26. Let $\theta: \mathbb{Z}_{2} \times \mathbb{Z}_{2 m} \rightarrow \operatorname{Diff}(M(b, d))$ be an action on the prism manifold $M(b, d)$ where $m \geq 2$. Then $\theta$ is primitive if and only if either $m=2^{n}$, or if $p$ is any odd prime divisor of $m$, then $d=0$ ( $\mathrm{mod}$ p).

Proof. We may assume that $\theta=\beta_{2}$, and therefore $\left.\beta_{2}\right|_{\mathbb{Z}_{2 m}}=\alpha_{2}$. Suppose that $\beta_{2}$ is primitive. This implies that $\alpha_{2}$ is primitive and the result follows from Theorem 25. Now suppose that either $m=2^{n}$, or if $p$ is an odd prime divisor of $m$, then $d=0(\bmod p)$. Note that this implies by Theorem 25 that $\alpha_{2}$ is primitive. Let $H$ be a subgroup of $\mathbb{Z}_{2} \times \mathbb{Z}_{2 m}$ and $\beta=\left.\beta_{2}\right|_{H}$. If

$H \cap\left(Z_{2} \times\{1\}\right) \neq 1$, then $M(b, d) / \beta$ is not a manifold. So we may assume that $H \cap\left(Z_{2} \times\{1\}\right)=1$, and hence $H$ is a subgroup of $\mathbb{Z}_{2 m}$. Since $\alpha_{2}$ is primitive, $M(b, d) / \beta$ is not a manifold showing that $\beta_{2}$ is primitive.

Proposition 27. Let $\theta: \mathbb{Z}_{2} \times \mathbb{Z}_{2} \rightarrow \operatorname{Diff}(M(b, d))$ be an action on the prism manifold $M(b, d)$. Then $\theta$ is primitive if $\theta$ is equivalent to either $\gamma_{5}, \beta_{6}$ or $\alpha_{7}$. If $\theta$ is equivalent to $\beta_{2}$ or $\beta_{5}$, then $\theta$ is primitive if and only if $b=0 \quad(\bmod 2)$.

Proof. If $\theta$ is either $\gamma_{5}, \beta_{6}, \alpha_{7}, \beta_{2}$ or $\beta_{5}$, then any subgroup of $\mathbb{Z}_{2} \times \mathbb{Z}_{2}$ restricted to a Heegaard Klein bottle $K$ is a product of the following homeomorphisms where $[r u] \in K:[r u] \rightarrow\left[r u e^{\pi i}\right],[r u] \rightarrow[r \bar{u}]$, and $[r u] \rightarrow\left[\frac{1}{r} u\right]$. The only fixed-point free action on $K$ is the homeomorphism $[r u] \rightarrow\left[\frac{1}{r} u e^{\pi i}\right]$. By definition of $\gamma_{5}, \beta_{6}$ or $\alpha_{7}$, they do not contain this homeomorphism, and hence they are primitive. But the actions $\beta_{2}$ and $\beta_{5}$ do contain this $Z_{2}$ subgroup and we obtain a covering $M(b, d) \rightarrow M(b, d) / \mathbb{Z}_{2}=\mathcal{O}_{1}(b, 2 d)$. Since $\mathcal{O}_{1}(b, 2 d)$ is a manifold if and only if 2 does not divides $b$, the result follows.

Proposition 28. Let $\theta: \operatorname{Dih}\left(\mathbb{Z}_{m}\right) \rightarrow \operatorname{Diff}(M(b, d))$ be an action on the prism manifold $M(b, d)$ for $m>2$.

1) If $\theta$ is equivalent to either $\alpha_{4}$ or $\alpha_{5}$, then $\theta$ is primitive if and only if for every prime divisor $p$ of $m$, $b=0 \quad(\bmod p)$.

2) If $\theta$ is equivalent to $\beta_{5}$, then $\theta$ is primitive if and only if $b$ is even and for every odd prime divisor $p$ of 
$m, d=0(\bmod p)$.

3) If $\theta$ is equivalent to either $\gamma_{5}, \delta_{5}, \alpha_{6}$ or $\alpha_{7}$, then $\theta$ is primitive if and only if either $m=2^{n}$, or if $p$ is an odd prime divisor of $m$, then $d=0(\bmod p)$.

4) If $\theta$ is equivalent to $\beta_{6}$, then $\theta$ is primitive if and only if either $m=2$ or, if for each odd prime divisor $p$ of $m, d=0(\bmod p)$.

Proof. We may assume $\theta$ is either $\alpha_{4}$ or $\alpha_{5}$, and thus $m=2 n+1$ and $\left.\theta\right|_{\mathbb{Z}_{m}}=\alpha_{1}$. If $\theta$ is primitive, then $\left.\theta\right|_{\mathbb{Z}_{m}}=\alpha_{1}$ is primitive, and the result follows by Theorem 25. For the converse, suppose that $H$ is a subgroup of $\operatorname{Dih}\left(\mathbb{Z}_{m}\right)=\mathbb{Z}_{m}{ }^{\circ}{ }_{-1} \mathbb{Z}_{2}$. If $H \cap \mathbb{Z}_{2} \neq 1$, then $M(b, d) / H$ is not a manifold. So we may assume $H \cap \mathbb{Z}_{2}=1$, and thus $H$ is a subgroup of $\mathbb{Z}_{m}$. Hence $H=\mathbb{Z}_{1}$ for some $l$ with $l$ dividing $m$. By Theorem 25, $\alpha_{1}$ is primitive, and so $M(b, d) / H$ is not a manifold. Thus $\alpha_{4}$ and $\alpha_{5}$ are primitive. The proof for the other cases is similar to Theorem 25.

Proposition 29. Let

$$
\theta: \operatorname{Dih}\left(\mathbb{Z}_{2} \times \mathbb{Z}_{2 m}\right) \rightarrow \operatorname{Diff}(M(b, d))
$$

be an action on the prism manifold $M(b, d)$.

1) If $m \geq 2$, then $\theta$ is primitive if and only if either $m=2^{n}$, or if $p$ is any odd prime divisor of $m$, then $d=0 \quad(\bmod p)$.

2) If $m=1$, then $\theta$ is primitive if and only if $b=0$ $(\bmod 2)$.

Proof. We may assume that $\theta=\beta_{7}$. Since

$\left.\beta_{7}\right|_{\mathbb{Z}_{2} \times \mathbb{Z}_{2 m}}=\beta_{2}$, using Propositions 26 and 27, a proof similar to that used in Proposition 28 proves the result.

\section{Lattice Structure}

In this section we compute the maximum length of a chain in the partially ordered sets $\mathcal{L}^{i}(\beta, \delta)$. In addition, we give necessary and sufficient conditions for $\mathcal{D}^{1}(\beta, \delta)$ to be a Boolean algebra.

Theorem 30. Let $\left(b_{1}, d_{1}\right)$ and $\left(b_{2}, d_{2}\right)$ be elements of $\mathcal{D}^{1}(\beta, \delta)$, and so $d_{i} \varepsilon_{i}=\delta$, where $\varepsilon_{i}$ is 1 or 2 for $i=1,2$. Suppose $\left(b_{2}, d_{2}\right) \geq\left(b_{1}, d_{1}\right)$. Then $b_{2}$ divides $b_{1}$ and $\varepsilon_{2} \geq \varepsilon_{1}$.

1) If $\varepsilon_{2}>\varepsilon_{1}$, then there exists $(b, d)$ in $\mathcal{D}^{1}(\beta, \delta)$ such that $\left(b_{2}, d_{2}\right)>(b, d)>\left(b_{1}, d_{1}\right)$.

2) If $\varepsilon_{1}=\varepsilon_{2}$, then $\frac{b_{1}}{b_{2}}$ is prime if and only if there does not exist $(b, d)$ in $\mathcal{D}^{1}(\beta, \delta)$ such that $\left(b_{2}, d_{2}\right)>$ $(b, d)>\left(b_{1}, d_{1}\right)$.

Proof. Since $\left(b_{1}, d_{1}\right)$ and $\left(b_{2}, d_{2}\right)$ are elements of $\mathcal{D}^{1}(\beta, \delta)$ for $i=1,2$, it follows that $\operatorname{g.c.d}\left\{b_{i}, d_{i}\right\}=1$, $b_{i}$ divides $\beta, \frac{\beta}{b_{i}}=1(\bmod 2)$, and $d_{i} \varepsilon_{i}=\delta$ where $\varepsilon_{i}=1$ or 2. By the definition of $\left(b_{2}, d_{2}\right) \geq\left(b_{1}, d_{1}\right)$, we have $b_{2} \mid b_{1}$ and $d_{2} \mid d_{1}$. Now $\frac{d_{1}}{d_{2}}=\frac{\varepsilon_{2}}{\varepsilon_{1}}$, which implies $\varepsilon_{2} \geq \varepsilon_{1}$

Suppose $\varepsilon_{2}>\varepsilon_{1}$. Thus $\varepsilon_{2}=2$ and $\varepsilon_{1}=1$, hence $2 d_{2}=d_{1}$. Now $\left(b_{1}, d_{2}\right) \in \mathcal{D}^{1}(\beta, \delta)$ and $\left(b_{2}, d_{2}\right)>\left(b_{1}, d_{2}\right)>\left(b_{1}, d_{1}\right)$ showing (1).

We now suppose that $\varepsilon_{1}=\varepsilon_{2}$, and thus $d_{1}=d_{2}$. Suppose there exists $(b, d)$ in $\mathcal{D}^{1}(\beta, \delta)$ such that $\left(b_{2}, d_{2}\right) \geq(b, d) \geq\left(b_{1}, d_{1}\right)$. It follows that $d_{1}=d=d_{2}$, $b_{2} \mid b$ and $b \mid b_{1}$. Therefore $\frac{b}{b_{2}} \cdot \frac{b_{1}}{b}=\frac{b_{1}}{b_{2}}$. Note that $\frac{b_{1}}{b_{2}}$ is prime if and only if either $(b, d)$ equals $\left(b_{2}, d_{2}\right)$ or $\left(b_{1}, d_{1}\right)$.

Corollary 31. Let $\left[\varphi_{1}\right]$ and $\left[\varphi_{2}\right]$ be elements of $\mathcal{L}^{\mathrm{l}}(\beta, \delta)$, such that $\varphi_{1}: \mathbb{Z}_{\varepsilon_{1} m_{1}} \rightarrow \operatorname{Diff}(M(b, d))$ and $\varphi_{2}: \mathbb{Z}_{\varepsilon_{2} m_{2}} \rightarrow \operatorname{Diff}\left(M\left(b_{2}, d_{2}\right)\right)$ where $m_{i}$ is odd and $\varepsilon_{i}$ is either 1 or 2 . Suppose $\left[\varphi_{2}\right]>\left[\varphi_{1}\right]$. Then $b_{2}$ divides $b_{1}$ and $\varepsilon_{2} \geq \varepsilon_{1}$.

1) If $\varepsilon_{2}=2$ and $\varepsilon_{1}=1$, then there exists $[\varphi] \in \mathcal{L}^{1}(\beta, \delta)$ such that $\left[\varphi_{2}\right]>[\varphi]>\left[\varphi_{1}\right]$.

2) If $\varepsilon_{1}=\varepsilon_{2}$ and $\frac{b_{1}}{b_{2}}$ is prime, there exists no $[\varphi] \in \mathcal{L}^{1}(\beta, \delta)$ such that $\left[\varphi_{2}\right]>[\varphi]>\left[\varphi_{1}\right]$.

Recall that the maximal element in $\mathcal{D}^{1}(\beta, \delta)$ is $\left(2^{n}, \delta\right)$ if $\delta$ is odd, and $\left(2^{n}, \delta / 2\right)$ if $\delta$ is even. To obtain the minimal element let $b_{0}$ be the largest odd divisor of $\beta$ such that g.c.d $\left\{b_{0}, \delta\right\}=1$. The minimal element is $\left(2^{n} b_{0}, \delta\right)$ if either $\delta$ is odd or if $n=0$, otherwise the minimal element is $\left(2^{n} b_{0}, \delta / 2\right)$.

Theorem 32. For the partially ordered sets $\mathcal{L}^{i}(\beta, \delta)$ where $1 \leq i \leq 7$ and $i \neq 3,6$, let $\beta=2^{n} p_{1}^{n_{1}} p_{2}^{n_{2}} \cdots p_{k}^{n_{k}}$ and $\delta=2^{m} p_{1}^{m_{1}} p_{2}^{m_{2}} \cdots p_{k}^{m_{k}}$ be the prime decompositions. Let $b_{0}$ be the largest odd divisor of $\beta$ relatively prime to $\delta$. Thus, $b_{0}=p_{1}^{l_{1}} p_{2}^{l_{2}} \cdots p_{k}^{l_{k}}$ where $l_{j}=0$ if and only if $\min \left\{n_{j}, m_{j}\right\}>0$ and $l_{j}=n_{j}$ if and only if $\min \left\{n_{j}, m_{j}\right\}=0$. Then the following chart gives the length of a maximum chain in each $\mathcal{L}^{i}(\beta, \delta)$.

\begin{tabular}{ccc}
\hline \multicolumn{3}{c}{ The maximum length of a chain } \\
\hline $\begin{array}{c}\text { Ordered set } \\
\mathcal{L}^{\mathrm{l}}(\beta, \delta), \mathcal{L}^{2}(\beta, \delta),\end{array}$ & Conditions & Max. length \\
$\mathcal{L}^{\urcorner}(\beta, \delta)$ & $\delta \neq 0 \quad(\bmod 2)$ & $\left(\sum_{i=1}^{k} l_{i}\right)+1$ \\
$\mathcal{L}^{\mathrm{l}}(\beta, \delta), \mathcal{L}^{2}(\beta, \delta)$, & $\delta=0 \quad(\bmod 2)$ & $\left(\sum_{i=1}^{k} l_{i}\right)+2$ \\
$\mathcal{L}^{\urcorner}(\beta, \delta)$ & $\operatorname{none}$ & $\left(\sum_{i=1}^{k} l_{i}\right)+1$ \\
$\mathcal{L}^{4}(\beta, \delta)$ & $\delta \neq 0 \quad(\bmod 2)$ and $n=0$ & $\left(\sum_{i=1}^{k} l_{i}\right)+1$ \\
$\mathcal{L}^{5}(\beta, \delta)$ & $\quad$ or $n \geq 1$ & $\left(\sum_{i=1}^{k} l_{i}\right)+2$ \\
$\mathcal{L}^{5}(\beta, \delta)$ & $\delta=0 \quad(\bmod 2)$ and $n=0$ &
\end{tabular}


Proof. We will consider first $\mathcal{L}^{1}(\beta, \delta)$. Since $\mathcal{L}^{1}(\beta, \delta)$ is isomorphic to $\mathcal{D}^{1}(\beta, \delta)$ we will prove the result for $\mathcal{D}^{1}(\beta, \delta)$. Suppose that $\beta$ and $\delta$ are both even, and thus $\left(b_{0}, \delta / 2\right)$ and $\left(2^{n}, \delta / 2\right)$ are the minimal and maximal elements of $\mathcal{D}^{1}(\beta, \delta)$ respectively. We may construct a chain in $\mathcal{D}^{1}(\beta, \delta)$

$$
\begin{aligned}
& \left(b_{0}, \delta / 2\right)<\left(b_{0} p_{1}^{-1}, \delta / 2\right)<\left(b_{0} p_{1}^{-2}, \delta / 2\right)<\cdots \\
& <\left(b_{0} p_{1}^{-l_{1}}, \delta / 2\right)<\cdots<\left(2^{n} p_{k}, \delta / 2\right)<\left(2^{n}, \delta / 2\right)
\end{aligned}
$$

such that dividing any two consecutive first coordinates yields a single prime in the prime decomposition of $b_{0}$. Now the length of this chain is $\left(\sum_{i=1}^{k} l_{i}\right)+1$. Since any maximal chain must contain both the minimal and maximal elements, it follows by the above theorem that this is a maximal chain. The other cases for $\mathcal{D}^{1}(\beta, \delta)$ are similar.

For the case $\mathcal{L}^{2}(\beta, \delta)$ note that $\mathcal{L}^{2}(\beta, \delta)$ is isomorphic to $\mathcal{D}^{2}(\beta, \delta)$, which by Theorem 16 is equal to $\cup_{j=1}^{m} \mathcal{D}^{1}\left(2^{m-j} b_{0}, \delta\right)$ if $\delta \neq 0(\bmod 2) ; \mathcal{D}^{1}\left(b_{0}, \delta\right)$ if $\delta=0$ $(\bmod 4)$; or $\cup_{j=1}^{m-1} \mathcal{D}^{1}\left(2^{m-j} b_{0}, \delta / 2\right) \cup \mathcal{D}^{1}\left(b_{0}, \delta\right)$ if $\frac{\delta}{2} \neq 0$ (mod 2). By Remark 17 each $\cup_{j=1}^{m} \mathcal{D}^{1}\left(2^{m-j} b_{0}, \delta\right)$ and $\cup_{j=1}^{m-1} \mathcal{D}^{1}\left(2^{m-j} b_{0}, \delta / 2\right)$ is a disjoint union of isomorphic lattices. The result now follows by applying the above $\mathcal{D}^{1}(\beta, \delta)$ case.

By Theorem $21, \mathcal{L}^{4}(\beta, \delta) \simeq \mathcal{D}_{*}^{1}(\beta, \delta)$. If $\delta$ is odd then $\mathcal{D}_{*}^{1}(\beta, \delta)=\mathcal{D}^{1}(\beta, \delta)$, and the result follows by the above. If $\delta$ is even, then a chain in $\mathcal{D}_{*}^{1}(\beta, \delta)$ can be constructed as the above where the second coordinate is replaced by $\delta$ proving this case also.

By Theorem 22, $\mathcal{L}^{5}(\beta, \delta)$ is isomorphic to $\cup_{j=0}^{m} \mathcal{D}^{1}\left(2^{j} b_{0}, \delta\right)$ if $\delta \neq 0(\bmod 2)$ and $m \geq 1 ; \mathcal{D}_{*}^{1}\left(b_{0}, \delta\right)$ if $\delta=0(\bmod 4)$ and $m \geq 1 ; \mathcal{D}^{1}\left(2^{m} b_{0}, \delta / 2\right) \cup \mathcal{D}_{*}^{1}(\beta, \delta)$ if $\frac{\delta}{2} \neq 0(\bmod 2)$ and $m \geq 1$; or $\mathcal{D}^{1}\left(b_{0}, \delta\right)$ if $m=0$. Using the above results, the first three cases give the maximum length of $\left(\sum_{i=1}^{k} l_{i}\right)+1$. When $m=0$, then there is no restriction on $\delta$. Thus the maximum length is $\left(\sum_{i=1}^{k} l_{i}\right)+1$ if $\delta$ is odd and $\left(\sum_{i=1}^{k} l_{i}\right)+2$ if $\delta$ is even.

For the remaining case, it follows by Theorem 24 that $\mathcal{L}^{7}(\beta, \delta) \simeq \mathcal{D}^{2}(\beta, \delta)$, which in turn is isomorphic to $\mathcal{L}^{2}(\beta, \delta)$. The result now follows by the above.

Theorem 33. For a pair of positive integers $\beta$ and $\delta$ with $\beta>\delta, \beta+\delta$ even, let $\frac{\beta+\delta}{2}=2^{m} \gamma$ where $\gamma$ is odd. Let $b_{0}$ be the largest odd divisor of $\gamma$ relatively prime to $d=\frac{\beta-\delta}{2}$ and let $\gamma=2^{s} p_{1}^{s_{1}} p_{2}^{s_{2}} \cdots p_{k}^{s_{k}}$ and $\delta=2^{t} p_{1}^{t_{1}} p_{2}^{t_{2}} \cdots p_{k}^{t_{k}}$ be their prime decompositions.
Thus, $b_{0}=p_{1}^{l_{1}} p_{2}^{l_{2}} \cdots p_{k}^{l_{k}}$ where $l_{j}=0$ if and only if $\min \left\{s_{j}, t_{j}\right\}>0$ and $l_{j}=s_{j}$ if and only if $\min \left\{s_{j}, t_{j}\right\}=0$. For the partially ordered sets $\mathcal{L}^{3}(\beta, \delta)$ and $\mathcal{L}^{6}(\beta, \delta)$ the maximum length of a chain is $\left(\sum_{i=1}^{k} l_{i}\right)+1$.

Proof. By Theorems 18 and 23 it follows that $\mathcal{L}^{3}(\beta, \delta)$ and $\mathcal{L}^{6}(\beta, \delta)$ are both isomorphic to $\mathcal{D}^{3}(\beta, \delta)$, which is equal to $\cup_{j=1}^{m} \mathcal{D}^{1}\left(2^{m-j} b_{0}, d\right)$ if $\beta-\delta \neq 0(\bmod 4)$ or $\mathcal{D}_{*}^{1}(\beta, \delta)$ if $\beta-\delta=0(\bmod 4)$. In both cases the maximum length is $\left(\sum_{i=1}^{k} l_{i}\right)+1$.

A lattice $L$ is a distributive lattice if for any $a, b, c$ in $L$,

$$
a \wedge(b \vee c)=(a \wedge b) \vee(a \wedge c) .
$$

Proposition 34. $\mathcal{D}^{1}(\beta, \delta)$ is a distributive lattice where for $\left(b_{1}, d_{1}\right)$ and $\left(b_{2}, d_{2}\right)$ in $\mathcal{D}^{1}(\beta, \delta)$ the join

$$
\left(b_{1}, d_{1}\right) \vee\left(b_{2}, d_{2}\right)=\left(\text { g.c.d }\left\{b_{1}, b_{2}\right\}, \min \left\{d_{1}, d_{2}\right\}\right)
$$

and the meet

$$
\left(b_{1}, d_{1}\right) \wedge\left(b_{2}, d_{2}\right)=\left(\text { l.c.m }\left\{b_{1}, b_{2}\right\}, \max \left\{d_{1}, d_{2}\right\}\right) .
$$

Proof. By Corollary $9, \mathcal{D}^{1}(\beta, \delta)$ is a lattice. A computation using the following equation

$$
\max \{l, \min \{m, n\}\}=\min \{\max \{l, m\}, \max \{l, n\}\}
$$

for any positive integers $l, m$ and $n$, shows that $\mathcal{D}^{1}(\beta, \delta)$ is a distributive lattice.

Remark 35. If we represent the minimal element by $\left(2^{n} b_{0}, \delta / \varepsilon\right)$ and the maximal element by $\left(2^{n}, \delta / \varepsilon\right)$ where $\varepsilon$ is either 1 or 2 , then for any element

$\left(2^{n} b, d\right) \in \mathcal{D}^{1}(\beta, \delta)$ we have

$$
\left(2^{n} b, d\right) \vee\left(2^{n} b_{0}, \delta / \varepsilon\right)=\left(2^{n} b, d\right)
$$

and

$$
\left(2^{n} b, d\right) \wedge\left(2^{n}, \delta / \varepsilon\right)=\left(2^{n} b, d\right) .
$$

A lattice $(B, \wedge, \vee)$ is said to be a Boolean algebra if the following hold:

1) $B$ is a distributive lattice having a minimal element 0 and a maximal element 1 .

2) For every $a \in B, a \vee 0=a$ and $a \wedge 1=a$.

3) For every $a \in B$ there exists $a^{\prime} \in B$ such that $a \vee a^{\prime}=1$ and $a \wedge a^{\prime}=0$.

Proposition 36. For the partially ordered set $\mathcal{D}^{1}(\beta, \delta)$ let $b_{0}$ be the largest odd divisor of $\beta$ such that g.c.d $\left\{b_{0}, \delta\right\}=1$ and let $b_{0}=\prod_{i=1}^{k} p_{i}^{l_{i}}$ be the prime decomposition. Then $\left(\mathcal{D}^{1}(\beta, \delta), \wedge, \vee\right)$ is a Boolean algebra if and only if $l_{i}=1$ for all $1 \leq i \leq k$.

Proof. Suppose that $l_{i}=1$ for all $1 \leq i \leq k$. By Remark 35 above and Proposition 34, it remains to show (3) of the definition. Let $\left(2^{n} b_{1}, d_{1}\right) \in \mathcal{D}^{1}(\beta, \delta)$. Now $2^{n} b_{1} \mid 2^{n} b_{0}$ 
and so let $b_{2}=\frac{b_{0}}{b_{1}}$. Observe that $\operatorname{g.c.d}\left\{b_{1}, b_{2}\right\}=1$ and l.c. $m\left\{b_{1}, b_{2}\right\}=b_{1} b_{2}=b_{0}$. If either $n \neq 0$ and $\delta$ is even, or if $\delta$ is odd, then all the elements in $\mathcal{D}^{1}(\beta, \delta)$ have the same second coordinate-either $\delta / 2$ in the first case or $\delta$ in the second case. In this case

$$
\left(2^{n} b_{1}, d_{1}\right) \wedge\left(2^{n} b_{2}, d_{1}\right)=\left(2^{n} b_{0}, d_{1}\right)
$$

and $\left(2^{n} b_{1}, d_{1}\right) \vee\left(2^{n} b_{2}, d_{1}\right)=\left(2^{n}, d_{1}\right)$. The remaining case is $n=0$ and $\delta$ even. If $d_{1}=\delta$ then let $d_{2}=\delta / 2$, and if $d_{1}=\delta / 2$ we let $d_{2}=\delta$. It follows that

$\left(2^{n} b_{1}, d_{1}\right) \wedge\left(2^{n} b_{2}, d_{2}\right)$ gives the minimal element and $\left(2^{n} b_{1}, d_{1}\right) \vee\left(2^{n} b_{2}, d_{2}\right)$ gives the maximal element.

We now suppose that $\left(\mathcal{D}^{1}(\beta, \delta), \wedge, \vee\right)$ is a Boolean algebra. Suppose there exists an $l_{j}>1$. The minimal element in $\mathcal{D}^{1}(\beta, \delta)$ is $\left(2^{n} b_{0}, \delta / \varepsilon\right)$ where $\varepsilon$ is either 1 or 2 . Now $\left(2^{n} b_{0} p_{j}^{-1}, \delta / \varepsilon\right)$ is an element of $\mathcal{D}^{1}(\beta, \delta)$ and $\left(2^{n} b_{0} p_{j}^{-1}, \delta / \varepsilon\right)>\left(2^{n} b_{0}, \delta / \varepsilon\right)$. There exists a complement $\left(2^{n} b, d\right)$ such that

$$
\left(2^{n} b, d\right) \wedge\left(2^{n} b_{0} p_{j}^{-1}, \delta / \varepsilon\right)=\left(2^{n} b_{0}, \delta / \varepsilon\right),
$$

and so l.c.m $\left\{b_{0} p_{j}^{-1}, b\right\}=b_{0}$. It follows that $p_{j}$ divides $b$. We also have $\left(2^{n} b, d\right) \vee\left(2^{n} b_{0} p_{j}^{-1}\right)$ equal to the maximal element $\left(2^{n}, \delta / \varepsilon\right)$, and so $g . c . d\left\{2^{n} b, 2^{n} b_{0} p_{j}^{-1}\right\}=2^{n}$. But since $l_{j}>1$, it follows that $p_{j}$ divides $b_{0} p_{j}^{-1}$, giving a contradiction.

Proposition 37. Let $I=\left(b_{0}, d_{0}\right) \downarrow$ be an ideal of a lattice $\mathcal{D}^{1}(\beta, \delta)$ such that $\left(b_{0}, d_{0}\right)$ is directly below $\left(2^{n}, d\right)$ which denotes the maximum element in the lattice. If $\left(b_{1}, d_{1}\right) \wedge\left(b_{2}, d_{2}\right) \in I$, then $\left(b_{1}, d_{1}\right) \in I$ or $\left(b_{2}, d_{2}\right) \in I$ and $I$ is a maximal ideal.

Proof. Let $\left(b_{1}, d_{1}\right) \wedge\left(b_{2}, d_{2}\right) \in I=\left(b_{0}, d_{0}\right) \downarrow$. Suppose both $\left(b_{i}, d_{i}\right) \notin I$ for $i=1,2$. Since there is no element between $\left(b_{0}, d_{0}\right)$ and $\left(2^{n}, d\right)$, we have

$\left(b_{i}, d_{i}\right) \vee\left(b_{0}, d_{0}\right)=\left(2^{n}, d\right)$, where g.c. $d\left(b_{i}, b_{0}\right)=2^{n}$ for $i=1,2$. This says that $b_{i}$ and $b_{0}$ do not have a common odd prime divisor for $i=1,2$.

On the other hand, $\left(b_{1}, d_{1}\right) \wedge\left(b_{2}, d_{2}\right) \leq\left(b_{0}, d_{0}\right)$ so that $b_{0} \mid$ l.c. $m\left\{b_{1}, b_{2}\right\}$. Since $b_{0}$ and $b_{i}$ do not have any common odd prime divisors, this forces $b_{0}=2^{n}$. As $\left(b_{0}, d_{0}\right)=\left(2^{n}, d_{0}\right)$ is not the maximum element, $d_{0}=2 d$. This result is possible only when the second coordinate is allowed to have an even number, otherwise it would be contradiction. Note that the second coordinate is an even number so that we must have $n=0$, and hence $\left(b_{0}, d_{0}\right)=(1,2 d)$. In addition, both $b_{1}$ and $b_{2}$ must be odd numbers. Now, $\left(b_{1}, d_{1}\right) \wedge\left(b_{2}, d_{2}\right) \leq\left(b_{0}, d_{0}\right)$ implies $d_{0}=2 d$ should divide $\max \left\{d_{1}, d_{2}\right\}$. It follows that at least one of $d_{i}$ must be equal to $2 d$. We may assume $d_{1}=2 d$ and thus $\left(b_{1}, d_{1}\right)=\left(b_{1}, 2 d\right)$. Since $b_{1}$ is an odd number and $b_{0}=1$, this shows $\left(b_{1}, d_{1}\right) \leq\left(b_{0}, d_{0}\right)$ telling us $\left(b_{1}, d_{1}\right) \in I$, which is a contradiction.

Remark 38. The converse of Proposition 37 is false. For example, consider $\mathcal{D}^{1}(45,11) . \quad(9,11) \downarrow$ is a prime ideal but not maximal.

Corollary 39. Let $I=\left(b_{0}, d_{0}\right) \downarrow$ be an ideal of a lattice $\mathcal{D}^{1}(\beta, \delta)$ such that $\left(b_{0}, d_{0}\right)$ is directly below $\left(2^{n}, d\right)$ which denotes the maximum element in the lattice. Let $L=\{0,1\}$ be a lattice where the partial ordering on $L$ is defined by $0 \leq 1$. Then the following are true and equivalent.

1) $I$ is a prime ideal.

2) $\mathcal{D}^{1}(\beta, \delta)-I$ is a prime filter.

3) There is a homomorphism $\phi: D^{1}(\beta, \delta)-I$ with $I=\phi^{-I}(0)$.

Proof. Condition (1) follows by Proposition 37, and conditions (2) and (3) follow by lattice theory (see for example [5]).

Proposition 40. Let $I=\left(b_{0}, d_{0}\right) \downarrow$ be an ideal of a lattice $\mathcal{D}^{1}(\beta, \delta)$ and $\left(2^{n}, d\right)$ denotes the maximum element in the lattice. Suppose that if $\left(b_{1}, d_{1}\right) \wedge\left(b_{2}, d_{2}\right) \in I$, then $\left(b_{1}, d_{1}\right) \in I$ or $\left(b_{2}, d_{2}\right) \in I$. If $\mathcal{D}^{1}(\beta, \delta)$ is a Boolean algebra, then I is maximal and $\left(b_{0}, d_{0}\right)$ is directly below $\left(2^{n}, d\right)$.

Proof. Since $\mathcal{D}^{1}(\beta, \delta)$ is a Boolean algebra, $I$ is maximal. If $\left(b_{0}, d_{0}\right) \leq\left(b^{\prime}, d^{\prime}\right)<\left(2^{n}, d\right)$, then $\left(b_{0}, d_{0}\right) \downarrow \subseteq\left(b^{\prime}, d^{\prime}\right) \downarrow$. This shows $\left(b_{0}, d_{0}\right)=\left(b^{\prime}, d^{\prime}\right)$.

\section{Group Lattice Structure}

Let $m$ and $n$ be relatively prime integers with $n>1$. Define the group $\pi(m, n)$ to be

$$
\pi(m, n)=\left\langle x, y \mid y x y^{-1}=x^{-1}, y^{2 m} x^{n}=1\right\rangle .
$$

Let $V$ and $W$ denote a solid torus and a twisted I-bundle over the Klein bottle $K$ respectively. Recall that the prism manifold $M(m, n)=V \cup_{\psi} W$, where $\partial V$ is identified to $\partial W$ by a homeomorphism $\psi: \partial V \rightarrow \partial W$ defined by $\psi(u, v)=\left(u^{s} v^{m}, u^{t} v^{n}\right)$, where $s$ and $t$ are integers satisfying $s n-t m=-1$. The fundamental group of $M(m, n)$ is $\pi(m, n)$.

Theorem 41. Let $H$ be a normal subgroup of $\pi(m, n)$. Then, either $H$ is cyclic or $H$ is isomorphic to $\pi(b, d)$ for some relatively prime integers $b$ and $d$ satisfying the following conditions: $b$ divides $m, \frac{m}{b}=1(\bmod 2), d=n$ and $\pi(m, n) / H=\mathbb{Z}_{m / b}$, or $2 d=n$ and $\pi(m, n) / H=\mathbb{Z}_{2 m / b}$. Furthermore, there exists a realizable isomorphism $\phi$ of $\pi(m, n)$ such that if $d=n$ then $\phi(H)=\left\langle x, y^{\frac{m}{b}}\right\rangle$, 
and if $2 d=n$ then $\phi(H)=\left\langle x^{2}, y^{\frac{m}{b}}\right\rangle$.

Proof. Let $H$ be a normal subgroup of $\pi(m, n)$. Let $v: M \rightarrow M(m, n)$ be the regular covering corresponding to $H$. Choose a component $\tilde{W}$ of $v^{-1}(W)$ and let $v_{0}=\left.v\right|_{\tilde{W}} \tilde{W}: \rightarrow W$. Since $W$ is a twisted I-bundle over a Klein bottle $K$ and $v_{0}: \tilde{W} \rightarrow W$ is a covering space, it follows that $\tilde{W}$ is either $T \times I$ where $T$ is a torus or a twisted $I$-bundle over a Klein bottle. Note that each component of $v^{-1}(V)$ is a solid torus. If $\tilde{W}$ is $T \times I$, then there are two components of $v^{-1}(V)$ whose boundaries are being identified with $\partial(T \times I)$, and thus $M$ is a lens space. In this case $\pi_{1}(M)=H$ is cyclic. If $\tilde{W}$ is a twisted $I$-bundle over the Klein bottle, then there is only one component of $v^{-1}(V)$ whose boundary is being identified with $\partial \tilde{W}$, and hence $M$ is a prism manifold. In this case $M=M(b, d)$ for some relatively prime integers $b$ and $d$. Furthermore there is a group action $G$ on $M(b, d)$ such that $M(b, d) / G=M(m, n)$. Now $v^{-1}(K)$ is a $G$-invariant Klein bottle. Hence by [4], the $G$-action is equivalent, via a homeomorphism $h$ of $M(b, d)$, to either a standard $\mathbb{Z}_{2 r+1}$-action with $m=(2 r+1) b$ and $n=d$, or a standard $\mathbb{Z}_{2(2 r+1)}$-action with $m=(2 r+1) b$ and $n=2 d$. These standard actions arise from the coverings of $M(m, n)$ corresponding to the subgroups $\left\langle x, y^{\frac{m}{b}}\right\rangle$ and $\left\langle x^{2}, y^{\frac{m}{b}}\right\rangle$ respecttively. Now $h$ projects to a homeomorphism of $M(m, n)$ realizing $\phi$.

Theorem 42. Let $\pi\left(b_{1}, d_{1}\right)=\left\langle x^{\varepsilon_{1}}, y^{\frac{m}{b_{1}}}\right\rangle$ and $\pi\left(b_{2}, d_{2}\right)=\left\langle x^{\varepsilon_{2}}, y^{\frac{m}{b_{2}}}\right\rangle$ be subgroups of $\pi(m, n)$ where $\varepsilon_{i}=1$ or 2 . Then

$$
\pi\left(b_{1}, d_{1}\right) \cap \pi\left(b_{2}, d_{2}\right)=\pi(b, d)=\left\langle x^{\max \left\{\varepsilon_{1}, \varepsilon_{2}\right\}}, y^{\frac{m}{b}}\right\rangle
$$

where $b=\operatorname{gcd}\left\{b_{1}, b_{2}\right\}$ and $d=\min \left\{d_{1}, d_{2}\right\}$. The group generated by $\pi\left(b_{1}, d_{1}\right)$ and $\pi\left(b_{2}, d_{2}\right)$ is

$\pi\left(b^{\prime}, d^{\prime}\right)=\left\langle x^{\min \left\{\varepsilon_{1}, \varepsilon_{2}\right\}}, y^{\frac{m}{b^{\prime}}}\right\rangle$ where $b^{\prime}=$ l.c.m $\left\{b_{1}, b_{2}\right\}$ and $d^{\prime}=\max \left\{d_{1}, d_{2}\right\}$.

Proof. Let $b=\operatorname{gcd}\left\{b_{1}, b_{2}\right\}$. Note that we have

$$
\begin{gathered}
\left(y^{\frac{m}{b_{i}}}\right)^{\frac{b_{i}}{b}}=y^{\frac{m}{b}} \text { for } i=1,2 . \text { This shows that } \\
\pi(b, d)=\left\langle x^{\max \left\{\varepsilon_{1}, \varepsilon_{2}\right\}}, y^{\frac{m}{b}}\right\rangle
\end{gathered}
$$

is a subgroup of $\pi\left(b_{1}, d_{1}\right) \cap \pi\left(b_{2}, d_{2}\right)=H$. Since $H$ contains $\pi(b, d)$, it follows that $H$ is not cyclic. By Theorem $41, H$ is isomorphic to $\pi(l, n)$ or $\pi(l, n / 2)$. Furthermore, $b$ divides $l$ and $l$ divides $b_{i}$, and since $b=$ g.c. $\left\{\left\{b_{1}, b_{2}\right\}\right.$, it follows that $b=l$. If $d_{1}$ or $d_{2}$ is $n / 2$, then since $H$ is a subgroup of $\pi\left(b_{i}, d_{i}\right)$, it follows by the above Theorem 41 that $H \simeq \pi(l, n / 2)$. Since $\pi(b, d)$ is a subgroup of $H$, we must have $d=n / 2$ showing $\pi(b, d)=H$. We now suppose $d_{1}=d_{2}=n$, and thus $d=n$ and $H \simeq \pi(l, n)$. It follows that $\pi(b, d)=H$.

Let $J$ be the group generated by $\pi\left(b_{1}, d_{1}\right)$ and $\pi\left(b_{2}, d_{2}\right)$. Now $x^{\min \left\{\varepsilon_{1}, \varepsilon_{2}\right\}}$ is clearly a generator of $J$ and $\pi\left(b^{\prime}, d^{\prime}\right)$. Since $\left(y^{\frac{m}{b^{\prime}}}\right)^{\frac{b^{\prime}}{b_{i}}}=y^{\frac{m}{b_{i}}}$, we have $J$ contained in $\pi\left(b^{\prime}, d^{\prime}\right)$. To show $\pi\left(b^{\prime}, d^{\prime}\right)$ is contained in $J$, we use the easily verifiable equation g.c.d $\left\{\frac{m}{b_{1}}, \frac{m}{b_{2}}\right\}$ l.c. $m\left\{b_{1}, b_{2}\right\}=m$, and by using $b^{\prime}=$ l.c. $m\left\{b_{1}, b_{2}\right\}$ we have g.c.d $\left\{\frac{m}{b_{1}}, \frac{m}{b_{2}}\right\}=\frac{m}{b^{\prime}}$.

Since there exist integers $s$ and $t$ such that $\frac{m}{b_{1}} s+\frac{m}{b_{2}} t=\frac{m}{b^{\prime}}$, we obtain $\left(y^{\frac{m}{b_{1}}}\right)^{s}\left(y^{\frac{m}{b_{2}}}\right)^{t}=y^{\frac{m}{b^{\prime}}}$ proving the result.

Let $\mathcal{S}(m, n)$ be the collection of subgroups $\pi\left(b, \frac{n}{\varepsilon}\right)=\left\langle x^{\varepsilon}, y^{\frac{m}{b}}\right\rangle$ of $\pi(m, n)$ where $\varepsilon=1$ or 2 .

Theorem 43. $\mathcal{S}(m, n)$ is a lattice of subgroups, and there exists a lattice isomorphism $\mathcal{S}(m, n) \rightarrow \mathcal{D}^{1}(m, n)$ which sends an element $\pi(b, d)$ in $\mathcal{S}(m, n)$ to the element $(b, d)$ in $\mathcal{D}^{1}(m, n)$.

Proof. If $\pi\left(b_{1}, d_{1}\right)$ and $\pi\left(b_{2}, d_{2}\right)$ are elements in $\mathcal{S}(m, n)$, define $\pi\left(b_{1}, d_{1}\right) \leq \pi\left(b_{2}, d_{2}\right)$ if $\pi\left(b_{2}, d_{2}\right)$ is a subgroup of $\pi\left(b_{1}, d_{1}\right)$. For $\pi\left(b_{1}, d_{1}\right)$ and $\pi\left(b_{2}, d_{2}\right)$ in $\mathcal{S}(m, n)$, define

$$
\pi\left(b_{1}, d_{1}\right) \vee \pi\left(b_{2}, d_{2}\right)=\pi\left(b_{1}, d_{1}\right) \cap \pi\left(b_{2}, d_{2}\right)
$$

and $\pi\left(b_{1}, d_{1}\right) \wedge \pi\left(b_{2}, d_{2}\right)$ to be the group generated by $\pi\left(b_{1}, d_{1}\right)$ and $\pi\left(b_{2}, d_{2}\right)$. By the above Theorem 42, $\mathcal{S}(m, n)$ is a lattice. Furthermore, the map which sends an element $\pi(b, d)$ in $\mathcal{S}(m, n)$ to the element $(b, d)$ in $\mathcal{D}^{1}(m, n)$ is a lattice isomorphism.

Corollary 44. $\mathcal{S}(m, n)$ is a distributive lattice, which is a Boolean algebra if and only if the prime decomposition of $m$ is $2^{j} \prod_{i=1}^{k} p_{i}$.

Proof. This follows by Propositions 34 and 36 and Theorem 43. 
For the following propositions write $m=p^{k} m_{0}$ where $p$ is an odd prime relatively prime to $m_{0}$.

Proposition 45. Let $\pi\left(p^{l} b, \frac{n}{\varepsilon}\right)$ and $\pi\left(p b, \frac{n}{\varepsilon}\right)$ be subgroups of $\pi(m, n)$ where $l \geq 1$. There exists a surjection $\psi_{l}: \pi\left(p^{l} b, \frac{n}{\varepsilon}\right) \rightarrow \pi\left(p b, \frac{n}{\varepsilon}\right)$.

Proof. Since $\pi\left(p^{l} b, \frac{n}{\varepsilon}\right)=\left\langle x^{\varepsilon}, y^{\frac{m}{p^{l^{b}}}}\right\rangle$ and $\pi\left(p b, \frac{n}{\varepsilon}\right)=\left\langle x^{\varepsilon}, y^{\frac{m}{p b}}\right\rangle$, define a function

$$
\psi_{l}: \pi\left(p^{l} b, \frac{n}{\varepsilon}\right) \rightarrow \pi\left(p b, \frac{n}{\varepsilon}\right)
$$

by $\quad \psi_{l}\left(x^{\varepsilon}\right)=x^{\varepsilon} \quad$ and $\quad \psi_{l}\left(y^{\frac{m}{p^{l} b}}\right)=y^{\frac{m}{p b}}$. Clearly $\psi_{l}$ preserves the first relation in $\pi\left(p^{l} b, \frac{n}{\varepsilon}\right)$. To show that $\psi_{l}$ preserves the second relation, it suffices to show that $y^{2 p^{l-1} m} x^{n}=1$. Write $p^{l-1}=2 s+1$, and note that

$$
y^{2 p^{l-1} m}=\left(y^{2 m}\right)^{2 s+1}=\left(y^{2 m}\right)^{2 s}\left(y^{2 m}\right)=\left(y^{4 m}\right)^{s} y^{2 m}=y^{2 m},
$$

since $y^{4 m}=1$. Thus $y^{2 p^{l-1} m} x^{n}=y^{2 m} x^{n}=1$, showing that $\psi_{l}$ is a homomorphism. Since $\psi_{l}$ takes generators to generators, it is also a surjection.

Proposition 46. Let $\pi\left(p^{l_{2}} b_{2}, \frac{n}{\varepsilon_{2}}\right) \leq \pi\left(p^{l_{1}} b_{1}, \frac{n}{\varepsilon_{1}}\right)$ be subgroups of $\pi(m, n)$ where $l_{1} \geq l_{2} \geq 1$. There exist surjections $\psi_{l_{i}}: \pi\left(p^{l_{i}} b_{i}, \frac{n}{\varepsilon_{i}}\right) \rightarrow \pi\left(p b_{i}, \frac{n}{\varepsilon_{i}}\right)$ for $i=1,2$ and a homomorphism $\theta: \pi\left(p b_{1}, \frac{n}{\varepsilon_{1}}\right) \rightarrow \pi\left(p b_{1}, \frac{n}{\varepsilon_{1}}\right)$, such that the following diagram commutes where $\tilde{v}$ and $v$ are inclusions:

$$
\begin{gathered}
\pi\left(p^{l_{2}} b_{2}, \frac{n}{\varepsilon_{2}}\right) \stackrel{\psi_{l_{2}}}{\longrightarrow} \pi\left(p b_{2}, \frac{n}{\varepsilon_{2}}\right) \\
\downarrow \tilde{v} \\
\pi\left(p^{l_{1}} b_{1}, \frac{n}{\varepsilon_{1}}\right) \stackrel{\psi_{l_{1}}}{\rightarrow} \pi\left(p b_{1}, \frac{n}{\varepsilon_{1}}\right) \stackrel{\theta}{\leftarrow} \pi\left(p b_{1}, \frac{n}{\varepsilon_{1}}\right)
\end{gathered}
$$

Proof. Let

$$
\pi\left(p^{l_{2}} b_{2}, \frac{n}{\varepsilon_{2}}\right)=\left\langle x^{\varepsilon_{2}}, y^{\frac{m}{p^{l_{2}} b_{2}}}\right\rangle \subset\left\langle x^{\varepsilon_{1}}, y^{\frac{m}{p^{l_{1}} b_{1}}}\right\rangle \pi\left(p^{l_{1}} b_{1}, \frac{n}{\varepsilon_{1}}\right) .
$$

Note that $b_{2}$ divides $b_{1}, \varepsilon_{2} \leq \varepsilon_{1}$ and $l_{2} \leq l_{1}$. By Proposition 45, there exist surjections

$$
\psi_{l_{i}}: \pi\left(p^{l_{i}} b_{i}, \frac{n}{\varepsilon_{i}}\right) \rightarrow \pi\left(p b_{i}, \frac{n}{\varepsilon_{i}}\right)
$$

defined by $\psi_{l_{i}}\left(x^{\varepsilon_{i}}\right)=x^{\varepsilon_{i}}, \psi_{l_{i}}\left(y^{\frac{m}{p^{p^{b_{i}}}}}\right)=y^{\frac{m}{p b_{i}}}$. Define a function $\theta: \pi\left(p b_{1}, \frac{n}{\varepsilon_{1}}\right) \rightarrow \pi\left(p b_{1}, \frac{n}{\varepsilon_{1}}\right)$ by $\theta\left(x^{\varepsilon_{1}}\right)=x^{\varepsilon_{1}}$ and $\theta\left(y^{\frac{m}{p b_{1}}}\right)=\left(y^{\frac{m}{p b_{1}}}\right)^{p^{l}}$ where $l=l_{1}-l_{2}$. Let $x^{\varepsilon_{1}}=c_{0}$ and $y^{\frac{m}{p b_{1}}}=c_{1}$, and note that the relations in this group are $c_{1} c_{0} c_{1}^{-1}=c_{0}^{-1}$ and $c_{1}^{2 p b_{1}} c_{0}^{\frac{n}{\varepsilon_{1}}}=1$. Write $p^{l}=2 s+1$ and observe that

$$
\left(c_{1}^{2 p b_{1}}\right)^{p^{l}}=\left(c_{1}^{2 p b_{1}}\right)^{2 s+1}=\left(c_{1}^{4 p b_{1}}\right)^{s}\left(c_{1}^{2 p b_{1}}\right)=c_{1}^{2 p b_{1}},
$$

since $c_{1}^{4 p b_{1}}=1$. Therefore

$$
\theta\left(c_{1}^{2 p b_{1}} c_{0}^{\frac{n}{\varepsilon_{1}}}\right)=\left(c_{1}^{2 p b_{1}}\right)^{p^{l}} c_{0}^{\frac{n}{\varepsilon_{1}}}=c_{1}^{2 p b_{1}} c_{0}^{\frac{n}{\varepsilon_{1}}}=1 .
$$

Clearly $\theta$ preserves the other relation, showing that $\theta$ is a homomorphism.

Since $p^{l_{2}} b_{2}$ divides $p^{l_{1}} b_{1}$ and $\varepsilon_{2}$ divides $\varepsilon_{1}$, it follows that the inclusion homomorphisms $\tilde{v}$ and $v$ are defined as follows: $\tilde{v}\left(x^{\varepsilon_{2}}\right)=\left(x^{\varepsilon_{1}}\right)^{\frac{\varepsilon_{2}}{\varepsilon_{1}}}$ and

$$
\tilde{v}\left(y^{\frac{m}{p^{2} b_{2}}}\right)=\left(y^{\frac{m}{p^{1} b_{1}}}\right)^{\frac{p^{l^{4}} b_{1}}{p^{2} b_{2}}},
$$

$v\left(x^{\varepsilon_{2}}\right)=\left(x^{\varepsilon_{1}}\right)^{\frac{\varepsilon_{2}}{\varepsilon_{1}}}$ and

$$
v\left(y^{\frac{m}{p b_{2}}}\right)=\left(y^{\frac{m}{p b_{1}}}\right)^{\frac{b_{1}}{b_{2}}} .
$$

One can easily check that

$$
\psi_{l_{1}} \circ \tilde{v}\left(x^{\varepsilon_{2}}\right)=\left(x^{\varepsilon_{1}}\right)^{\frac{\varepsilon_{2}}{\varepsilon_{1}}}=\theta \circ v \circ \psi_{l_{2}}\left(x^{\varepsilon_{2}}\right)
$$

and

$$
\psi_{l_{1}} \circ \tilde{v}\left(y^{\frac{m}{p^{2} b_{2}}}\right)=y^{\frac{p^{(l-1)} m}{b_{2}}}=\theta \circ v \circ \psi_{l_{2}}\left(y^{\frac{m}{p^{l_{2} b_{2}}}}\right),
$$

which verifies that our diagram commutes. 
Proposition 47. $\mathcal{S}\left(\mathrm{pm}_{0}, n\right)$ is a sublattice of $\mathcal{S}\left(p^{k} m_{0}, n\right)$, and there exists a lattice surjection $\Psi: \mathcal{S}\left(p^{k} m_{0}, n\right) \rightarrow \mathcal{S}\left(p m_{0}, n\right)$ induced by the the family of group homomorphisms $\left\{\psi_{l}\right\}$ such that $\Psi$ restricted to $\mathcal{S}\left(\mathrm{pm}_{0}, n\right)$ is the identity.

Proof. It is clear that $\mathcal{S}\left(p m_{0}, n\right)$ is a sublattice of $\mathcal{S}\left(p^{k} m_{0}, n\right)$. If $\pi(b, d) \in \mathcal{S}\left(p^{k} m_{0}, n\right)$, then $b=p^{l} b^{\prime}$ for some $l \leq k$. By Proposition 45, there exists a surjection $\psi_{l}: \pi\left(p^{l} b^{\prime}, \frac{n}{\varepsilon}\right) \rightarrow \pi\left(p b^{\prime}, \frac{n}{\varepsilon}\right)$. Define $\Psi\left(\pi\left(p^{l} b^{\prime}, \frac{n}{\varepsilon}\right)\right)=\pi\left(p b^{\prime}, \frac{n}{\varepsilon}\right)$. By the commutative diagram in Proposition 46, it follows that $\Psi$ is order preserving.

Theorem 48. Let $m=2^{j} \prod_{i=1}^{k} p_{i}^{m_{i}}$ be the prime decomposition. Then $\mathcal{S}\left(2^{j} \prod_{i=1}^{k} p_{i}, n\right)$ is a sublattice of $\mathcal{S}\left(2^{j} \prod_{i=1}^{k} p_{i}^{m_{i}}, n\right)$, and there exists a lattice surjection

$$
\Psi: \mathcal{S}\left(2^{j} \prod_{i=1}^{k} p_{i}^{m_{i}}, n\right) \rightarrow \mathcal{S}\left(2^{j} \prod_{i=1}^{k} p_{i}, n\right)
$$

induced by a family of group homomorphisms such that $\Psi$ restricted to $\mathcal{S}\left(2^{j} \prod_{i=1}^{k} p_{i}, n\right)$ is the identity.

Proof. Apply

$$
\Psi_{r}: \mathcal{S}\left(2^{j} p_{r}^{m_{r}} \prod_{1 \leq i \leq k}^{i \neq r} p_{i}^{m_{i}}, n\right) \rightarrow \mathcal{S}\left(2^{j} p_{r} \prod_{1 \leq i \leq k}^{i \neq r} p_{i}^{m_{i}}, n\right)
$$

repeatedly defined in Proposition 47 for $1 \leq r \leq k$ to obtain the result where $\Psi$ is the compositions of those $\Psi_{r}$ 's.

\section{Some Examples}

In this section we present several examples which illustrate the main theorems.

Example 49. $\mathcal{O}_{1}(315,14)$. This example illustrates Theorem 12 that $\mathcal{L}^{1}(315,14)$ is isomorphic to $\mathcal{D}^{1}(315,14)$.

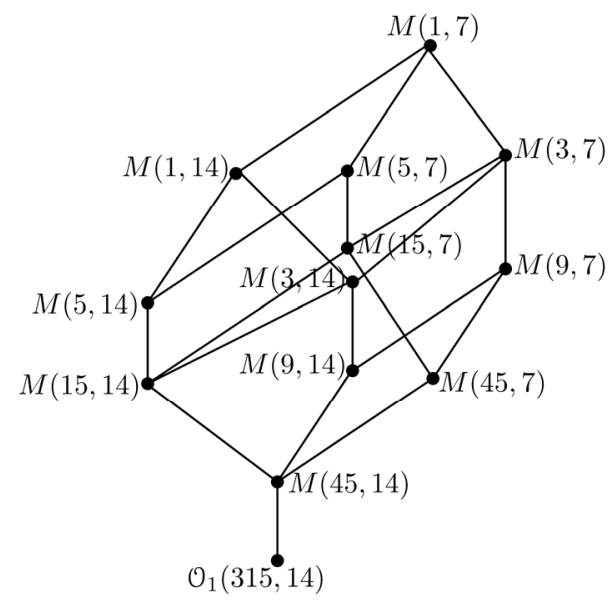

Example 50. $\mathcal{O}_{1}(1155,11)$. This is a Boolean lattice/ algebra by Proposition 36 since $1155=3 \times 5 \times 7 \times 11$.

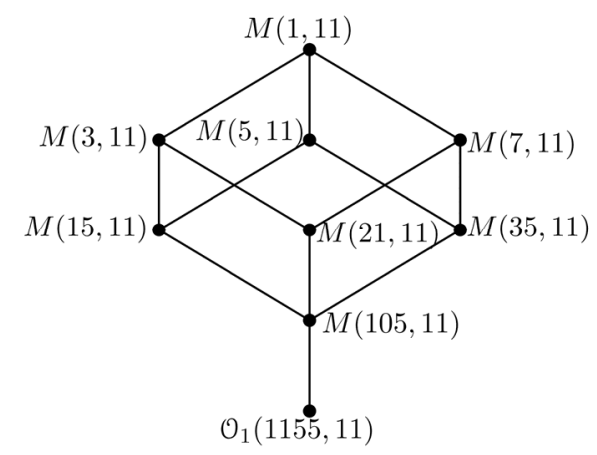

Prime ideals are: $(3,11) \downarrow,(5,11) \downarrow$ and $(7,11) \downarrow$ Their complements are lters which are: $(35,11) \uparrow$, $(21,11) \uparrow$ and $(15,11) \uparrow$ respectively.

Example 51. $\mathcal{O}_{2}(126,20)$. Since $\delta=20=0(\bmod 4)$, this example illustrates Theorems 15 and 16 that $\mathcal{L}^{2}(126,20) \simeq \mathcal{D}^{2}(126,20) \simeq \mathcal{D}^{1}(63,20)$.

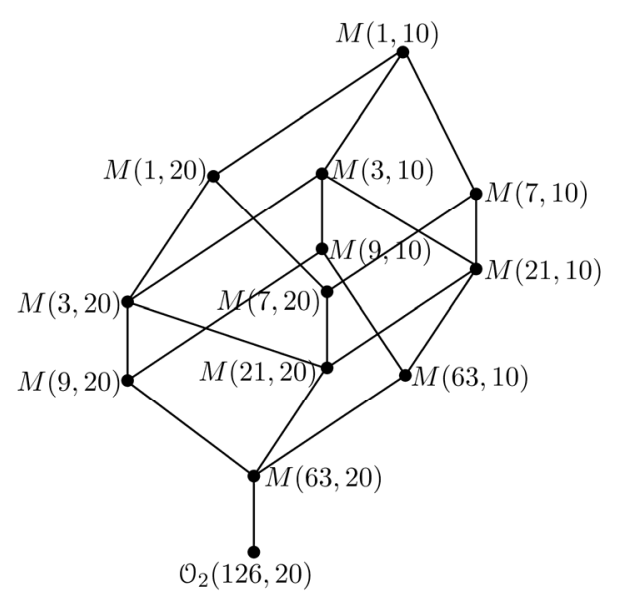

Example 52. $\mathcal{O}_{2}(5040,20)$. This example again illustrates Theorem 16 and also that $\mathcal{D}^{2}(5040,20)$ is isomorphic to $\mathcal{D}^{2}(126,20)$.

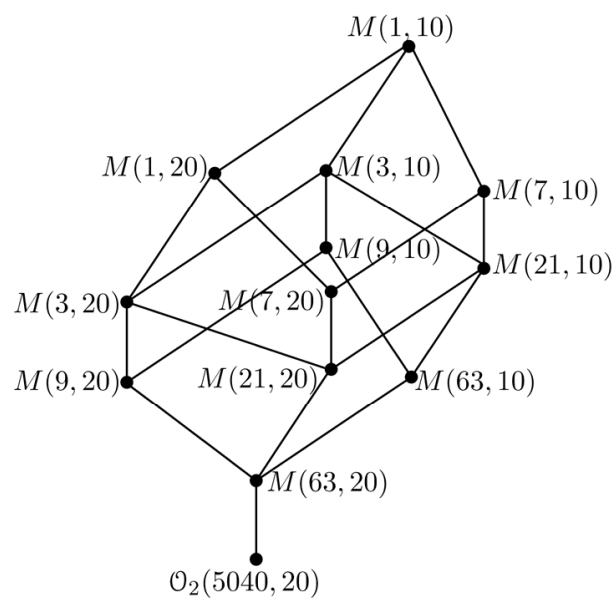


Example 53. $\mathcal{O}_{2}(5040,5)$. This illustrates Theorem $16 \quad$ isomorphic lattices that $\mathcal{D}^{2}(5040,5)$ is isomorphic to a disjoint union of $\quad \mathcal{D}^{1}(63,5) \cup \mathcal{D}^{1}(126,5) \cup \mathcal{D}^{1}(252,5) \cup \mathcal{D}^{1}(504,5)$.

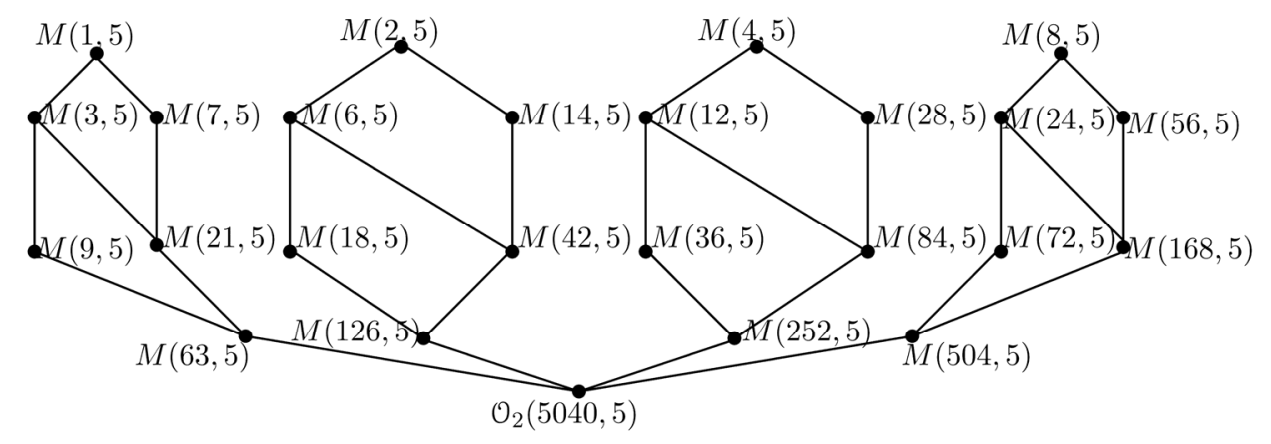

Example 54. $\mathcal{O}_{2}(5040,10)$. This example illustrates Theorem 16 that $\cup_{j=1}^{3} \mathcal{D}^{1}\left(2^{j} 63,5\right) \cup \mathcal{D}^{1}(63,10)$.

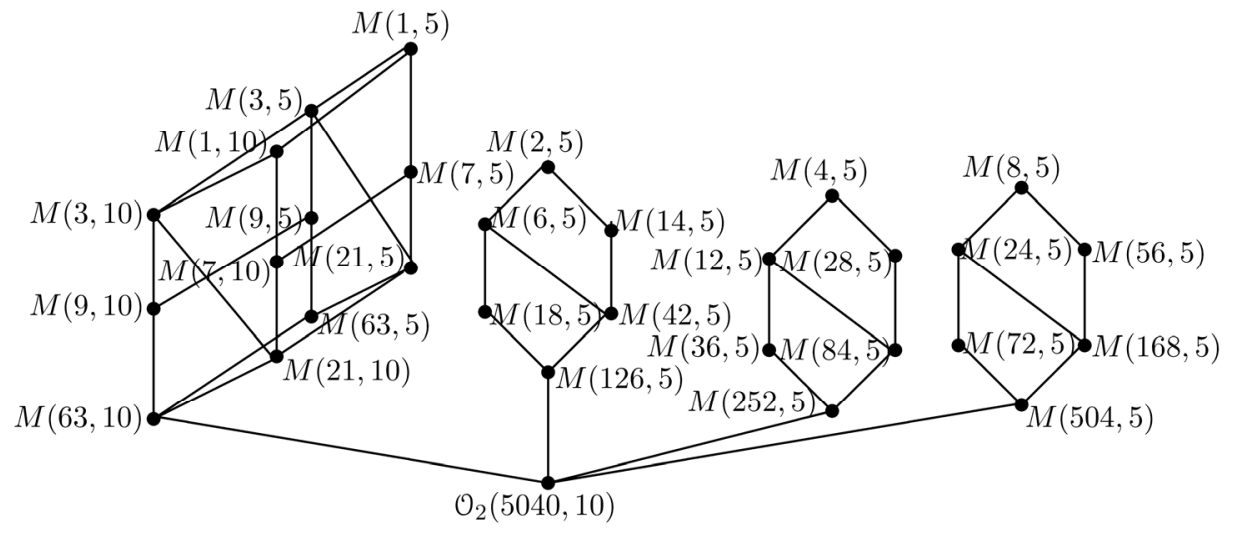

Example 55. $\mathcal{O}_{3}(5030,10)$. This example illustrates

Theorems 18 and 19 that

$$
\mathcal{L}^{3}(5030,10) \simeq \mathcal{D}^{3}(5030,10) \simeq \mathcal{D}_{*}^{1}(63,2510) .
$$

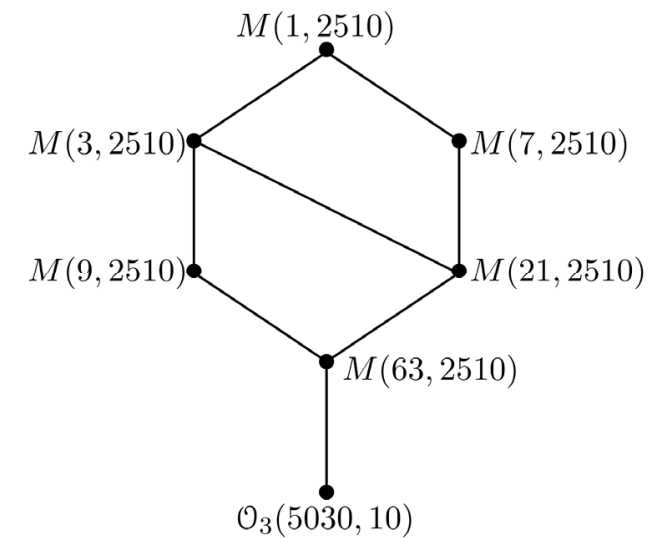

Example 56. $\mathcal{O}_{4}(126,5)$. This example illustrates Theorem 21 that

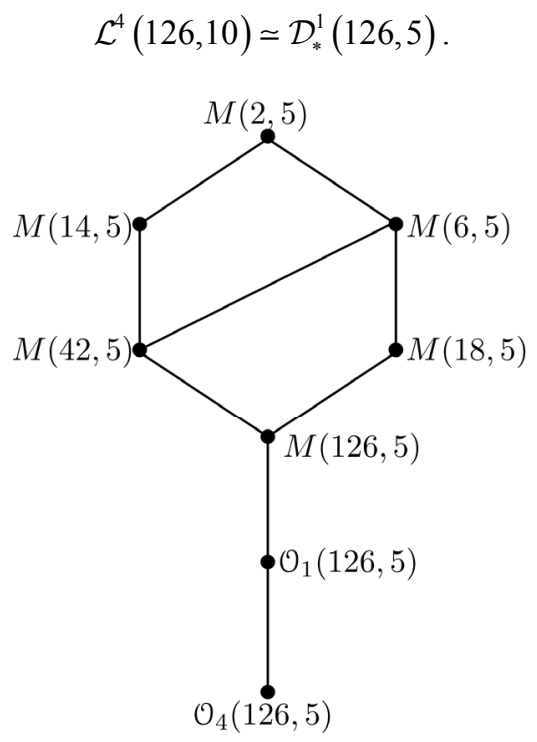

Example 57. $\mathcal{O}_{5}(630,5)$. This example illustrates Theorem 22 that

$$
\mathcal{L}^{5}(630,5) \simeq \mathcal{D}^{1}(63,5) \cup \mathcal{D}^{1}(126,5) .
$$




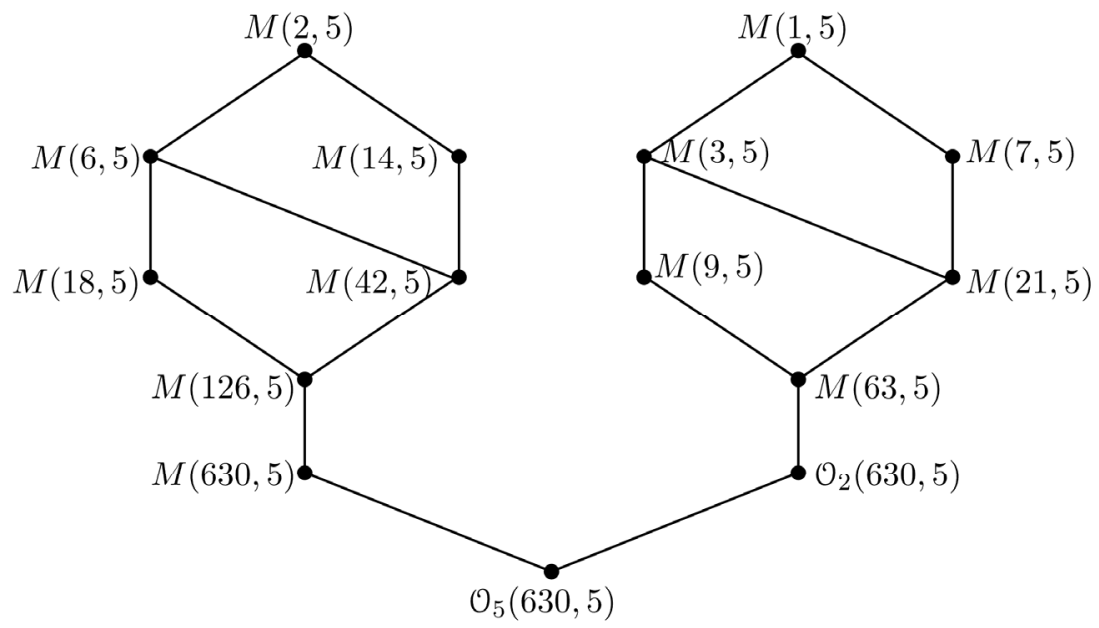

Example 58. $\mathcal{O}_{5}(5040,10)$. This example illustrates Theorem 22 that $\mathcal{L}^{5}(5040,10) \simeq \mathcal{D}^{1}(1008,5) \cup \mathcal{D}_{*}^{1}(5040,10)$.

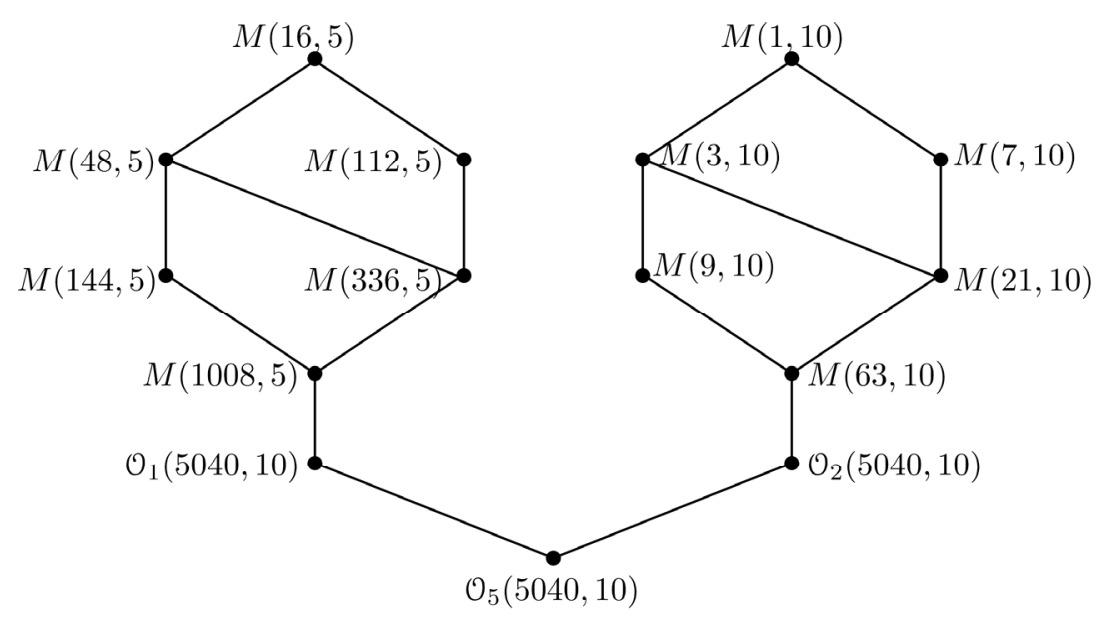

Example 59. $\mathcal{O}_{6}(63,5)$. This example illustrates Theorems 19 and 23 that

$$
\mathcal{L}^{6}(63,5) \simeq \mathcal{D}^{1}(17,29) \cup \mathcal{D}^{1}(34,29)
$$

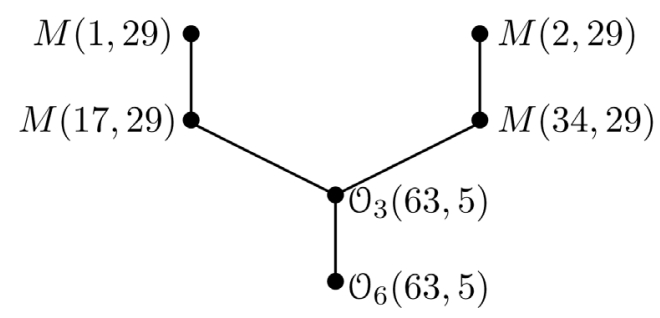

Example 60. $\mathcal{O}_{7}(126,20)$. This illustrates Theorems 16 and 24 that

$$
\mathcal{L}^{7}(126,20) \simeq \mathcal{D}^{2}(126,20) \simeq \mathcal{D}^{1}(63,20) .
$$

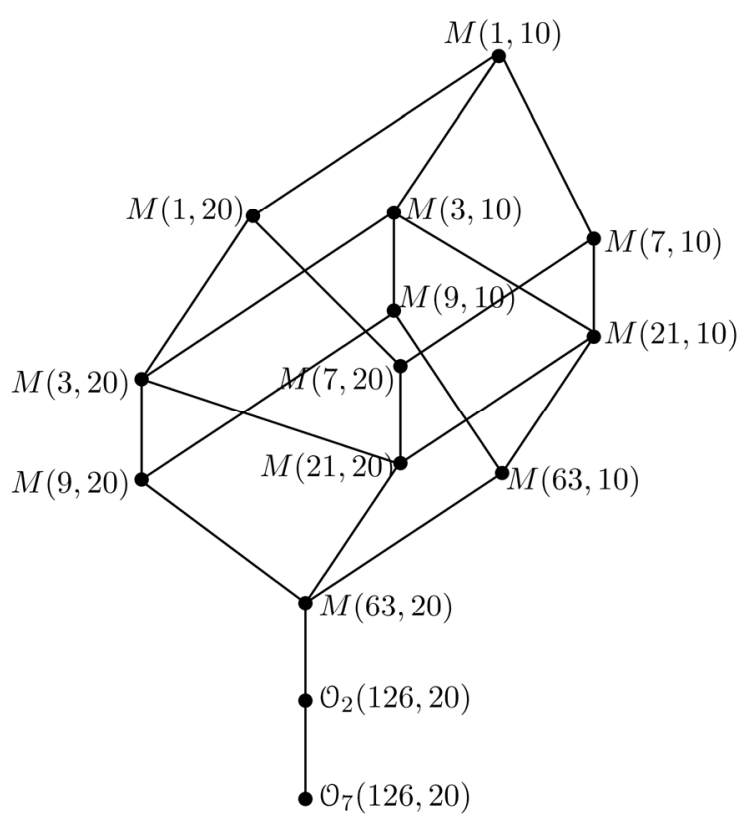


Example 61. This is an example of "crush" to illustrate Theorem 42.
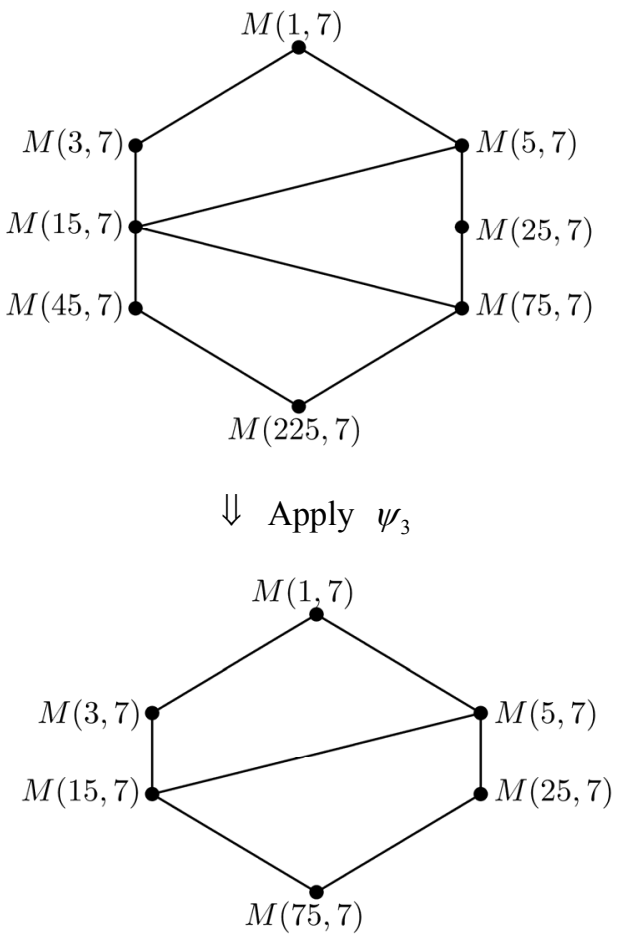

$\Downarrow$ Apply $\psi_{5}$

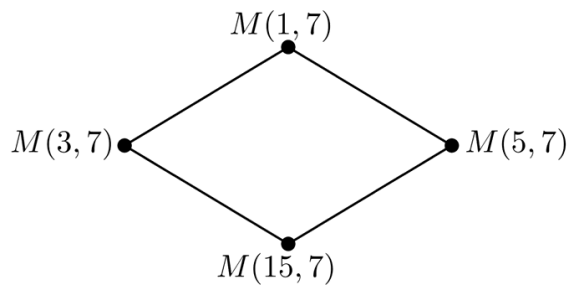

\section{REFERENCES}

[1] J. Kalliongis and A. Miller, "Orientation Reversing Actions on Lens Spaces and Gaussian Integers," Journal of Pure and Applied Algebra, Vol. 212, No. 3, 2008, pp. 652-667. doi:10.1016/j.jpaa.2007.06.022

[2] R. Stanley, "Enumerative Combinatorics Volume 1," Wadsworth \& Brooks/Cole, New York, 1986.

[3] R. Ohashi, "The Isometry Groups on Prism Manifolds, Dissertation," Saint Louis University, Saint Louis, 2005.

[4] J. Kalliongis and R. Ohashi, "Finite Group Actions on Prism Manifolds Which Preserve a Heegaard Klein Bottle," Kobe Journal of Math, Vol. 28, No. 1, 2011, pp. 6989.

[5] B. A. Davey and H. A. Priestley, "Introduction to Lattices and Order," Cambridge Mathematical Textbooks, Cambridge University Press, Cambridge, 1990. 